\title{
Computer Aided Constructivism
}

\author{
Brian R. Gaines \\ University of Victoria \\ gaines@uvic.ca
}

\author{
Mildred L. G. Shaw \\ University of Calgary \\ mildred@cpsc.ucalgary.ca
}

\section{Introduction}

George Kelly's early background, wartime experience and continuing interest in engineering, cybernetics and mathematics is evident in the systemic foundations he developed for personal construct psychology (PCP) that make it simple and natural to model through computer technology. He saw technology as providing essential support for humanistic psychology, remarking in a paper on methodology in the Journal of Humanistic Psychology that:

"It would, in my opinion, be a serious mistake for psychologists who hope to raise man from the position of an unwitting subject in an experiment to a posture of greater dignity to abandon technology. The spirit of man is not enlarged by withholding his tools. Just as it took the technology of gunpowder and the printing press to turn humanism into something more than classicism, so now it requires an appropriate technology for humanistic psychology to realize its objectives." (Kelly, 1969a, p.53).

Computers and related notions of automata, cybernetics and information processing had begun to play a role in psychological modeling in the 1950s (Quastler, 1955). Kelly was aware of this, noting in his book on PCP that:

"The practical task of reducing information to a form which can be handled by electronic computing machines has forced scientists to reconsider the mathematical structure of knowledge itself" (p.63-64) ${ }^{1}$.

He was an invited commentator at the first conference on Computer Simulation of Personality, remarking that:

"There are two major ways in which I see a computer simulation program contributing to an experimental science of psychology. First, it can simulate the logic of a theoretical system and, with a series of inputs, explicate the theory and its implications in ways the original theoretician would take a lifetime to figure out on his own. The second contribution can be to the processing of data provided by a given subject" (Kelly, 1963, p.227).

Kelly's first anticipation was validated by the development of interactive computer programs simulating psychologists and their subjects. For example, Weizenbaum's (1966) ELIZA program simulated the conversational interaction of a Rogerian therapist, by substituting phrases input by the user in the computer's responses. Shaw's (1978) PEGASUS program used similar techniques to emulate and enhance the conversational interaction of a personal construct psychologist eliciting a repertory grid. Colby, who had presented his research on computer modeling of neurosis at the same conference as Kelly, used Weizenbaum's techniques to program a computer

\footnotetext{
${ }^{1}$ Citations without a full reference are to Kelly (1955) The Psychology of Personal Constructs.
} 
to simulate a paranoiac patient (Colby, 1981). In the 1980s the expert system research community implemented Kelly's psychological theories to model and emulate human social and psychological processes as Kelly had anticipated (Shaw and Gaines, 1983; Boose, 1984; Gaines and Shaw, 1993).

Kelly's second anticipation was validated by the development of computer programs for the analysis of psychological data. In the context of PCP, for example, Slater's $(1976 ; 1977)$ Ingrid program applied Gower's (1966) geometric model of principal component analysis to Kelly's (1969b) notion of the psychological space represented in a repertory grid. Shaw's (1978) Focus program provided a hierarchical cluster analysis of repertory grid data in terms of the original grid so that the basis of the analysis was clearly apparent.

This chapter focuses on the role of computer-based tools and techniques in constructivist research. In the past thirty years the increasing capabilities and ubiquitous availability of personal computers connected to the Internet has led to them playing significant roles in all areas of psychological research. They support precisely controlled, complex interaction, powerful and rapid data analysis, the graphic presentation of such analysis in a comprehensible and compelling form, and conversational interaction enhancing human meaning making processes. Such powerful tools can be used to make a significant contribution to a research program when used well, but they can also be used to create a false impression of authoritative technique without due care and understanding in their use.

Our overall objective is to provide a framework for understanding how to use computer capabilities in a principled fashion to support constructivist research studies.

\section{What Constitutes a Constructivist Research Orientation in PCP?}

If we wish to ensure that computer technology is used effectively to support constructivist studies it is important to clarify what constitutes a constructivist method. Fortunately, Kelly was a methodologist par excellence and describes and discusses in depth the essential features of constructivist approaches not only to clinical psychology but also to a wide range of disciplines including the sciences and mathematics. His methodological framework was developed from that of Dewey whose pragmatic, instrumentalist analysis of human psychological processes has had a major influence on modern philosophy, psychology and educational practice, so that there is a rich ongoing literature consistent with and complementary to Kelly's writings (Rorty, 1982; Boisvert, 1988; Kulp, 1992; Shook, 2000).

Dewey and Kelly saw psychological processes as deriving from the future-orientation of living systems, the development of capabilities to be able to anticipate a world that had sufficient coherence in time for such anticipation to be reasonably effective and provide evolutionary advantage. Hume (1888) had noted that there is no logical rationale for it to be possible to anticipate future events, and hence it is an empirical phenomenon that the world we live in often exhibits patterns that enable future experience to be anticipated from past experience. As Dewey notes:

"While there is no a priori assurance that any particular instance of continuity will recur, the mind endeavors to regulate future experience by postulating recurrence. So far as the anticipation is justified by future events, the notion is confirmed. So far as it fails to work the assured continuity is dropped or corrected" (Dewey, 1911) 
In his widely circulated essay on the need for a recovery in philosophy, Dewey suggested that such anticipatory processes constituted what is meant by our notion of mind:

"Ability to anticipate future consequences and to respond to them as stimuli to present behavior may well define what is meant by a mind or by consciousness." (Dewey, 1917)

He associates anticipation based on experience with our notions of meaning:

"To be able to use the past to judge and infer the new and unknown implies that, although the past thing has gone, its meaning abides in such a way as to be applicable in determining the character of the new" (Dewey, 1933)

Kelly (pp.129, 154, 157) based his theoretical psychology on Dewey's insights, taking anticipation as the generative principle underlying all psychological phenomena, that "a person's processes are psychologically channelized by the ways in which he anticipates events" (p.46), and deriving all other aspects of psychological processes as corollaries of this fundamental postulate. His first corollary is that of construction, that "a person anticipates events by construing their replications" (p.50), where:

"By construing we mean 'placing an interpretation': a person places an interpretation upon what is construed. He erects a structure, within the framework of which the substance takes shape or assumes meaning. The substance which he construes does not produce the structure; the person does" (p.50)

Thus, for Kelly as for Dewey, the meaning making processes underlying our constructions of experience are an essential side effect of our use of past experience to anticipate aspects of current and potential experience. The principles of constructivist psychology derive from an analysis of what it is to be an anticipatory system, and constructivist studies need to look behind the constructions they model to the roles these constructions play in the anticipatory processes they support.

Methodological Principle 1: Meaning is ascribed to experience to make it comparable with other experience in order to anticipate further aspects of the experiences. Constructivist studies focus on meaning making processes. Computer support for such studies should be evaluated in terms of its enhancement of those processes.

Kelly's mode of exposition of PCP, as a postulate and its corollaries, follows that of Hull (1940) in his Mathematico-Deductive Theory of Rote Learning that Kelly admired, but reduces Hull's eighteen postulates to one. This is an instance of one of Kelly's most significant contributions, that he was minimalist in his theoretical system, introducing no more theoretical constructs than were necessary. He emphasizes this minimalism by not taking as fundamental many common constructs commonly adopted in theories of psychology:

"the term learning, so honorably embedded in most psychological texts, scarcely appears at all. That is wholly intentional; we are for throwing it overboard altogether. There is no ego, no emotion, no motivation, no reinforcement, no drive, no unconscious, no need." (p.x)

"Some writers have considered it advisable to try to distinguish between 'external' events and 'internal' events. In our system there is no particular need for making this kind 
of distinction. Nor do we have to distinguish so sharply between stimulus and response, between the organism and his environment, or between the self and the not-self." (p.55)

One may see this care to avoid unnecessary distinctions as a natural meta-consequence of a theory which emphasizes that each distinction one makes has significant consequences, and hence one should treat making a distinction as a major methodological issue.

Methodological Principle 2: Each construction used in meaning making processes can have significant consequences for the ensuing anticipations. Constructivist studies should be minimalist in the prior constructions brought to the study, making these constructions and their consequences for the study explicit. Computer support for the studies may introduce its own prior constructions that should be included in this process of explication.

Note that some prior assumptions are always necessary. One cannot commence a study with a completely empty meaning system. The methodological issues are that they should be made explicit and that they should not be multiplied unnecessarily. As Kelly notes:

"the knotty problems which sooner or later trick the scientist into intellectual contortions or into torturing his data with fancy statistical computations are likely to be traceable to the theory's fundamental postulate." (p.35)

It is also important to note that it is not possible for a study to investigate the validity of all its prior assumptions. As Kelly notes:

"we should bear in mind that the moment we question the truth of a statement proposed as a postulate, that statement is no longer a postulate in our subsequent discourse. A statement, therefore, is a postulate only if we accord it that status. If we bring the statement into dispute, as well we may in some instances, we must recognize that we are then arguing from other postulates either explicitly stated or, more likely, implicitly believed." (p.47)

Kelly provides many examples from both everyday life and scientific practice of the problems created by anticipations derived from constructions that, because they are implicitly postulated, are not recognized as the source of the problems.

Kelly used the term personal in describing his psychology to emphasize that meanings are idiosyncratic, but he also recognized that much individual activity is concerned with calibrating personal meanings against those of others:

"Those construction systems which can be communicated can be widely shared. The last half century has shown much progress in the development of ways of making personal constructs and construction systems more communicable. We have developed a scientific psychological vocabulary. A better way of saying this is that our public construction systems for understanding other people's personal constructs are becoming more precise and more comprehensive." (p.9)

Methodological Principle 3: Meanings are personal but communication requires inter-personal calibration which may not necessarily be effective. Constructivist studies need to investigate whether the use of the same terms reflects the same underlying distinction. Computer tools need to be able to support such investigation.

Kelly notes that one basis for the formation of public construction systems is shared experience: 
"Persons anticipate both public events and private events... No two people can play precisely the same role in the same event, no matter how closely they are associated... But does this mean that there can be no sharing of experience? Not at all; for each may construe the likenesses and differences between the events in which he himself is involved, together with those in which he sees that the other person is involved" (p.55)

Methodological Principle 4: Shared experiences provide a basis for calibrating and comparing personal constructs, but the notion of a shared experience is itself problematic. Constructivist studies based on the assumption that elements of experience are shared between individuals need to make that assumption explicit and, if possible, to test it. Computer tools that assume common elements of experience can be treated as identical may produce misleading results if that assumption is not valid.

The calibration of personal meanings with those of the community derives from anticipations that the individual should be able to communicate with that community, but is not a deterministic constraint. Individuals have other anticipations which may override such considerations, and Kelly expresses this in what is arguably his most important corollary:

"a person chooses for himself that alternative in a dichotomized construct through which he anticipates the greater possibility for the elaboration of his system" (Kelly, 1970, p.15).

This choice corollary underlies one of Kelly's most important notions, that of constructive alternativism, "there are always some alternative constructions available to choose among in dealing with the world" (p.15). He also notes that not all constructions are equally useful, "some of them are definitely poor implements" (p.15).

The choice corollary captures the essential boundary between logic and psychology, that there is an underlying logic to anticipatory processes which, as illustrated in the next section, computers can support and emulate. But there is also human choice in the way in that meanings are given to experience which is strongly influenced by the anticipations ensuing from that choice but not at all determined by them. As Kelly notes, this limits what computers can emulate:

"A sorting machine, no matter how complex, is not a thinking machine as long as we have to select data to feed into it" (p.64).

Harnad (1990) termed this issue the symbol grounding problem and studies of possible resolutions in both people and machines have become a major focus of psychological (Pecher and Zwaan, 2005) and artificial intelligence research (Taddeo and Floridi, 2005).

Methodological Principle 5: There is always choice in the meanings that are used to interpret experience. Constructivist studies need to recognize the choices being made and the anticipations underlying them. Computers can model the basis of choice but not necessarily the choice process itself.

One consequence of Kelly's systemic formulation of PCP is that it provides a model of any anticipatory system, not only individuals but also groups, organizations, disciplines, cultures, societies, and human civilization as a whole. There is no need to invoke controversial notions of group minds, since there is no invocation of notions of an individual mind as a causative structure. There is only the simple systemic principle that the anticipatory processes of any entity can be analyzed using the principles which Kelly develops. 
Methodological Principle 6: The anticipatory processes of people acting together may be modeled using the principles of PCP. Constructivist studies can encompass individuals, groups, organizations, disciplines, cultures, societies, and human civilization as a whole. Computers can support the complex data structuring and analysis required for such studies.

One of the groups involved in constructivist studies is the psychologist together with her or his subjects, and the principles of PCP apply equally to all involved. The anticipatory processes of the psychologist are similar to those of the subjects and need to be modeled as part of the study if they are not to be a source of undocumented artifacts.

Methodological Principle 7: The anticipatory processes of psychologists are similar to those of their subjects. Constructivist studies need to recognize that the meaning making processes of those conducting them are at least as great an influence on the outcome as those of the subjects being studied. Computer tools can be used to model the meaning processes of those conducting the study as well as those being studied and the interactions between them.

There are many more methodological principles and issues. We have chosen to highlight those above because they address many of the issues raised by users of our PCP-based computer programs. There is one further principle that has been a major one in the design of these programs, that there should be no 'magic' in the use of them:

Methodological Principle 8: Computer programs used in PCP studies should be designed to be supportive colleagues rather than authoritarian experts. The assumptions underlying them and the processes they carry out should be simple and understandable and relate as directly as possible to the principles of PCP.

The following sections illustrate how computational tools may be used to model and support Kelly's personal construct psychology.

\section{The Logical Structure of Construct Networks}

Kelly introduces his notion of a "construction system" with the following metaphor:

"Man looks at his world through transparent patterns or templets which he creates and then attempts to fit over the realities of which the world is composed. The fit is not always very good. Yet without such patterns the world appears to be such an undifferentiated homogeneity that man is unable to make any sense out of it. Even a poor fit is more helpful to him than nothing at all." (p.8-9)

Deconstructing the notion of a templet is a good starting point for a logical model of Kelly's constructivist theory. Dictionary definitions illustrate how the term captures some of his most important insights. "A model or standard for making comparisons," emphasizes the role of a psychological templet in enabling experiences to be compared for purposes of anticipation. "A pattern or gauge used as a guide in making something accurately," captures the role of a psychological templet in shaping experience, that something is modified to fit the templet. The fitting of a templet can be an active process of changing the world, not just a passive process of gauging whether the world fits the templet.

These connotations of the term, templet, nicely span the range of meanings that Kelly accommodates within the term, anticipation: of prediction of what may happen; of action to 
make something happen; of creative imagination of what might happen or be made to happen; and of preparation for eventualities that may well never happen.

\subsection{Logical Relations Between Templets}

We will take as primitive notions: experience, templet, and a relation between them of fit, that a templet may be said to fit an experience, and examine the possible relations between templets.

One possible relation is entailment, that if one templet fits an experience then it entails that another templet will also fit that experience. As a concrete example, consider the boundary of a building lot as a templet within which a house designer must fit a house. If a builder has given us a list of house designs that will fit on a lot we are considering and we then consider a larger lot whose boundary would encompass the original lot we know this entails that any house design we were considering will also fit the larger lot, and there may be additional house designs that will fit it also.

Another possible relation is incompatibility creating opposition or contrast, that if one templet fits an experience then another will not fit that experience. For example, if building lot templets have to be considered in three dimensions because some are on the side of a steep slope then it may be that the houses designed for flat lots are incompatible with sloping lots and vice versa.

Such relations between templets enable us to make anticipations from the fit of one templet to the fit of another. There are other bases for anticipation. For example, if we walk around part a finished housing project and notice that only the houses on the largest lots have double garages, we might anticipate that we will need to purchase a house on such a lot if we want that type of garage. That anticipation is based on matching against experience rather than an intrinsic relation between templets. We might be told this is the situation by the realtor rather than noticing it ourselves, in which case the experience is mediated rather than direct. We might find that the house designer is only prepared to put a triple garage house on the largest lots, which creates more complex entailment/contrast relations between templets than we had previously understood.

\subsection{Representing Logical Relations Between Templets}

These are the types of issues of relations between templets, experience and anticipation that Kelly encompasses in the corollaries to his fundamental postulate that elaborates his notion of a construct system and its central role in human psychology. We can express them in logical terms suitable for computer implementation by representing the entailment relation as an arrow from one templet to those that it entails (Fig.1 left), that if templet A fits an experience then templet B will fit; and the contrast relation by a negated arrow from one templet to those with which it is incompatible (Fig.1 center), that if templet A fits an experience then templet $\mathrm{C}$ will not fit.

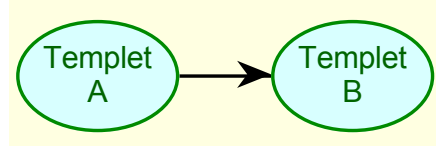

Entailment

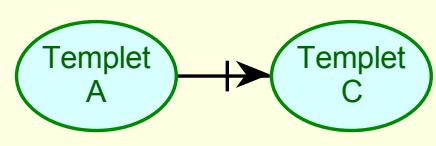

Incompatibility

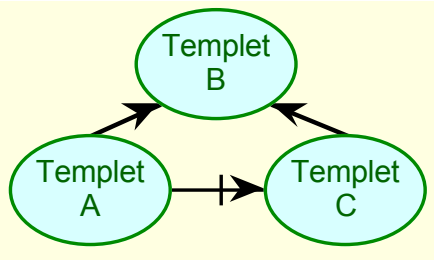

Construct

Figure 1 Some relations between templets 
These two relations enable us to represent Kelly's basic structure, that he terms a construct, as shown on the right of Figure 1 where templets $\mathrm{A}$ and $\mathrm{C}$ are incompatible and each entails templet B. Templet B captures the commonality between experiences that fall within the range of convenience of the construct, and the polar opposites (poles), $\mathrm{A}$ and $\mathrm{C}$, capture the contrast between them.

The arrows of entailment and contrast may be used to represent more elaborate networks of relations between templets. As Kelly noted, "no construct ever stands entirely alone; it makes sense only as it appears in a network" (p.304). Fig. 2 left gives a concrete example of a construct of size with poles large and small.
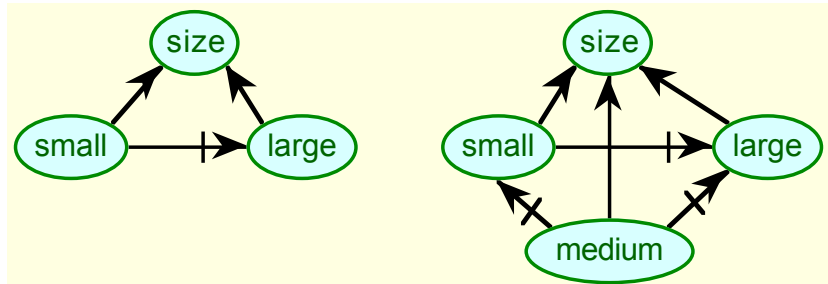

Figure 2 Some constructs

Fig. 3 right elaborates the network by adding the intermediate pole medium which is opposite to both small and large. Note that we can conceptualize this structure as a network of three linked constructs, each having the same range of convenience, or as a single construct having three poles. Multipolarity is a structure built on bipolarity. Note that we can also conceptualize these constructs as an attribute (size) having possible values (small, medium, large). Other literatures discuss constructs using different terminologies but describing the same logical structure.

\subsection{Hierarchies}

Fig. 3 left shows how each pole of the construct can be used as the range of convenience of a construct that makes finer distinctions. This structure is a hierarchy, a structure long used to classify knowledge, for example, in Aristotle's Categories and Roget's Thesaurus.
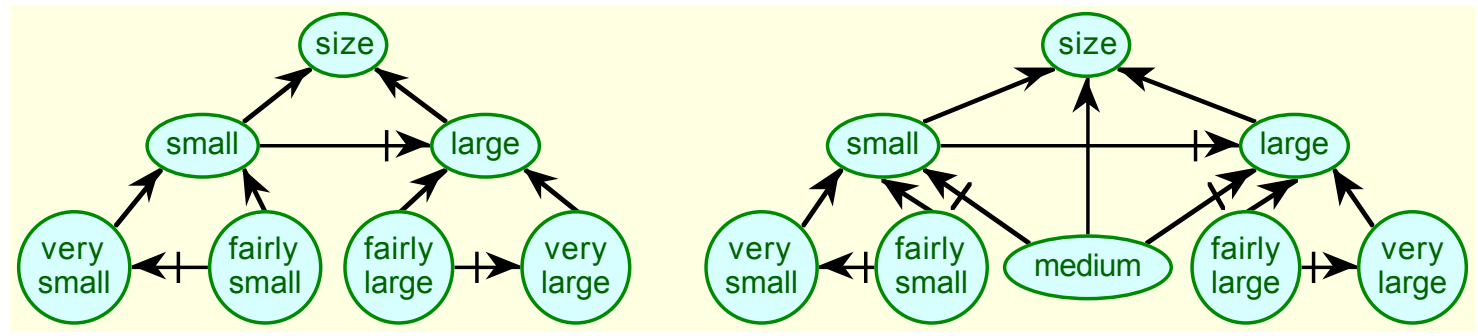

Figure 3 Some construct hierarchies

The network shown can be conceptualized as: three linked constructs; a single construct having four poles and internal structure; an attributes with values; or a hierarchy. It illustrates what Kelly (p.57) means when he notes that "one construct may subsume another as one of its elements." Fig.3 right shows how the intermediate pole can also be added.

\subsection{Rating Scales}

The obvious ordinal relationship between the poles of the construct networks in Figs. 2 and 3 can be derived from the logical structure by taking certain poles to be fundamental or salient and the 
others to derive their meanings from the salient poles they entail. For example, in Fig.2 right if small and large are taken as the salient poles then medium is characterized through its entailment that neither small nor large will fit. The sets of salient implications for each pole are:

$$
\text { small } \rightarrow \text { [small, not large }], \text { medium } \rightarrow \text { [not small, not large], large } \rightarrow \text { [not small, large }]
$$

The cardinalities of the set-theoretical symmetric differences between these sets of salient implications are:

$$
\delta(\text { small }, \text { medium })=2, \delta(\text { medium }, \text { large })=2, \delta(\text { small }, \text { large })=4
$$

which shows that the function, $\delta$, is a linear distance measure placing the poles along a threepoint rating scale.

A similar analysis of Fig.3 left and right gives rise to four- and five-point rating scales respectively if very small and very large are taken as additional salient poles. The value of 2 for the distances between the terminal pole suggests that there may be intermediate points with distance 1, and this is so. In Fig. 3 right small and large correspond to points intermediate between very small and fairly small or fairly large and very large, respectively, along a seven point scale. There are also two other intermediate points not shown that extend this to a ninepoint scale.

Thus, as Kelly (p.141-145) discusses in detail, some forms of construct network have a natural interpretation in terms of rating scales. Note, however, that different structures may generate the same scale, so that there is not necessarily an unambiguous logical representation of a rating scale. For example, Gaines (2009) analyzes a non-hierarchical construct network that also generates two- through nine-point scales. This ambiguity has been discussed in the PCP literature as the problem of determining the intended meaning of the center point of a rating scale (Yorke, 2001).

\subsection{Derived Entailment and Contrast Relations}

There are additional arrows of entailment and contrast that could be added to Fig. 3 but are not present because they can be derived from those already in place. For example, that the templet very small fits an experience entails that size fits that experience can be derived because very small entails small and small entails size. That very small contrasts with medium can be derived from very small implies small but medium contrasts with small.

The logical inference process involved in this derivation is very simple, consisting of propagating chosen and derived fits through the network. Such propagation is one of the basic algorithms of logical theorem provers (Davis, Logemann and Loveland, 1962) and also the mechanism of spreading activation in psychological models of semantic memory (Collins and Quillian, 1969).

Fig.4 shows an inference algorithm in the RepNet tool in Rep 5 (Gaines, 2009) being used to derive the fit of other templets when one templet has been chosen to fit an experience. A vertical bar is used to indicate fit, and a horizontal bar to indicate lack of fit. On the left it has been asserted that very small fits and the inference algorithm has used the arrows of entailment and contrast to derive that small also fits, fairly small does not, and so on. On the right medium fits has been asserted and the fit of the other templets derived. 

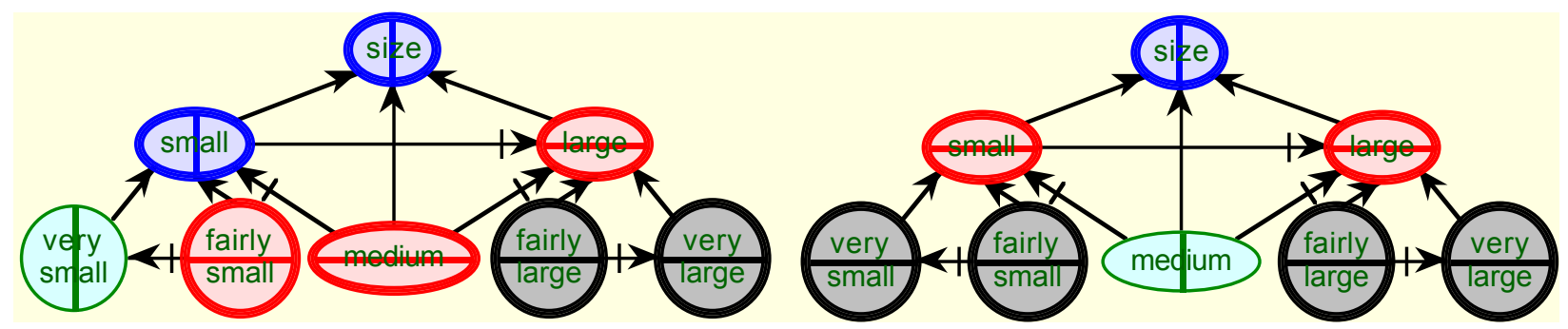

Figure 4 Derivation through inference in construct networks

\subsection{Relevance}

Kelly (p.63) notes that the tripartite structure of a construct distinguishes between lack of fit due to contrast and that due to irrelevance. In Fig.1 right, if we know that templet B does not fit an experience then we would not inquire about templets $\mathrm{A}$ and $\mathrm{C}$ because we would see them as irrelevant.

The reason they do not fit is because they are inapplicable rather than that they might possibly fit but do not. In the more concrete terms of Figs. 2 and 3, if the notion of size is not applicable to an element of experience then it is not relevant, or meaningless, to consider whether the element is large or small.

The management of our construing of experience in terms of relevance has obvious processing advantages in that we do not need to consider parts of the network that are irrelevant. Similar advantages appertain to inter-personal interaction where we do not need to communicate that which is irrelevant.

Much of social and organizational structure derives from the management of relevance. A baker does not need to have a construct network relevant to manufacturing machinery. A chemist does not need the repertoire of constructs of a philosopher or vice versa.

\subsection{Meta-values}

Note that Kelly has not introduced any non-standard logical notions in his model of relevance. A templet is either chosen to fit or it is not (or the choice is left open, but that is a deferral of choice, not a non-standard truth value). Relevance is managed through the structure of a construct. It is irrelevant to consider whether a templet fits an experience if the experience does not fall within the templet's associated range of convenience.

However, it may be useful in some situations to treat relevance as if it were an additional logical constant, that the fit between a construct pole and an experience may be true, false, or inapplicable, where inapplicable means 'false but irrelevant.' Kelly (p.315) discusses this in his chapter on the Structure of Psychological Space as the need for a third marker in a conceptual grid when the element falls outside the range of convenience of a construct.

We have found it useful in Rep 5 to support five meta-values: open meaning that an element has not been construed on a particular construct; unknown meaning that a construction cannot be supplied; any meaning that any pole might fit; none meaning that none of the defined poles fit; and inapplicable meaning that the construct is irrelevant. 


\section{More Complex Templet Structures Within Construct Networks}

It is tempting to look at the logical structure of construct poles and see them as predicates in standard predicate calculus. If the templets were logical predicates that could be applied to experience then the relations represented are ones that might hold among them. However, templets are generally rather more complex than logical predicates, encompassing logical formulae having structure, variables and relations between them. A templet may fit the same experience in more than one way, and in fitting it may instantiate its variables and do so in more than one way.

\subsection{Complex Templets}

Fig. 5 illustrates more complex templets through a hierarchy representing those a home decorator might used in discussion various options to a client. The notions of art object and furniture have a rich structure of connotations that would require a large construct network to represent but, in this context of use, the decorator can take those networks for granted and focus only on the distinctions relevant to the discussion represented in Fig. 5.

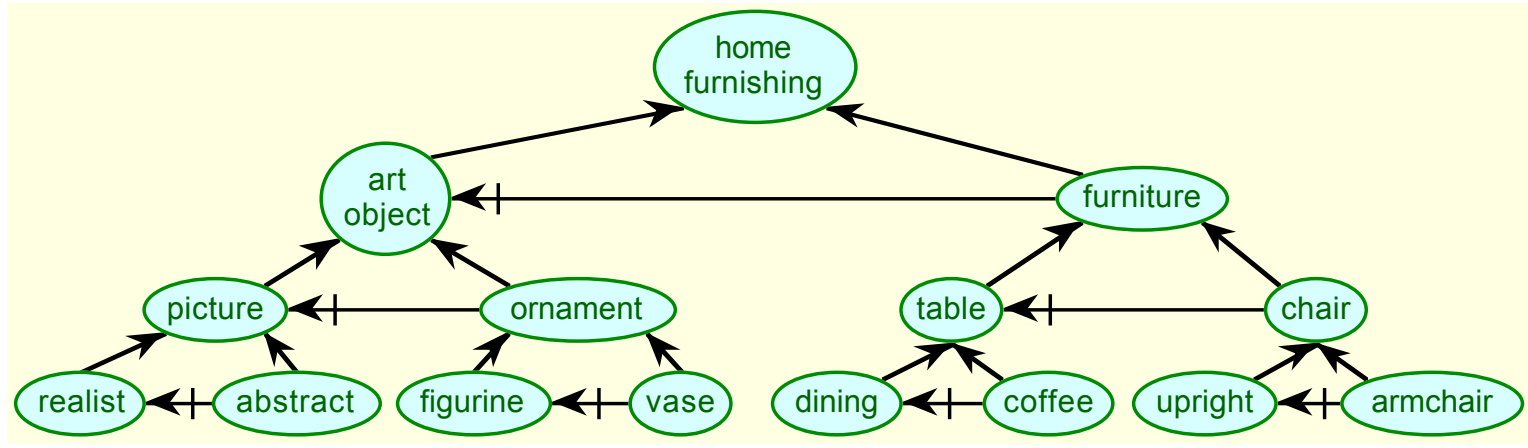

Figure 5 A hierarchy of more complex constructs

Thus, the network structure is phenomenological rather than definitional. Fitting an element to a templet forming one pole of a construct implies that it will not be fitted to the templet represented by the other pole and implies that some other templets in the network will or will not fit. These inferences follow from the entailments and contrasts for whatever reason they are present in someone's construct network. Their sources may be purely psychological, that someone happens to see the world in that way, or partially logical in that the connotations of the templets are such that they entail one another or contrast with one another.

Kelly (1955) provides a wide range of examples of how the constructs may arise, and it is important to realize that the entailments and contrasts involved may stem from many diverse sources, involving a variety of structures and processes.

\subsection{Representing the Connotations of Complex Templets}

To illustrate how the connotations of a complex templet are represented in a construct network, Fig.6 represents the notion of art object as a construct network derived from the art literature. Weitz (1977) argues that art objects are stereotypical examples of what he terms an open concept, subject to change and lacking formal definition. Danto (1964) had previously suggested a matrix structure for conceptualizing art objects in which the rows are conceptual dimensions, essentially constructs, the columns are art objects, and the number of rows increases as 
innovations in art invoke new dimensions of construction. Gaut (2000; 2005) develops such a matrix in detail to provide what he terms a cluster concept for an art object in which some but not all of the dimensions may be instantiated by particular art objects.

Fig.6 top shows the eleven constructs which Gaut presents as the relevant dimensions along which one characterizes an art object. At the center an art object is shown as entailing each of these dimensions, that is, as falling within their ranges of convenience. At the bottom a stereotypical ideal art object is shown as entailing the positive pole of each dimension. This construct network provides a conceptual framework for much of the discussion in the aesthetics community about the nature of art objects.

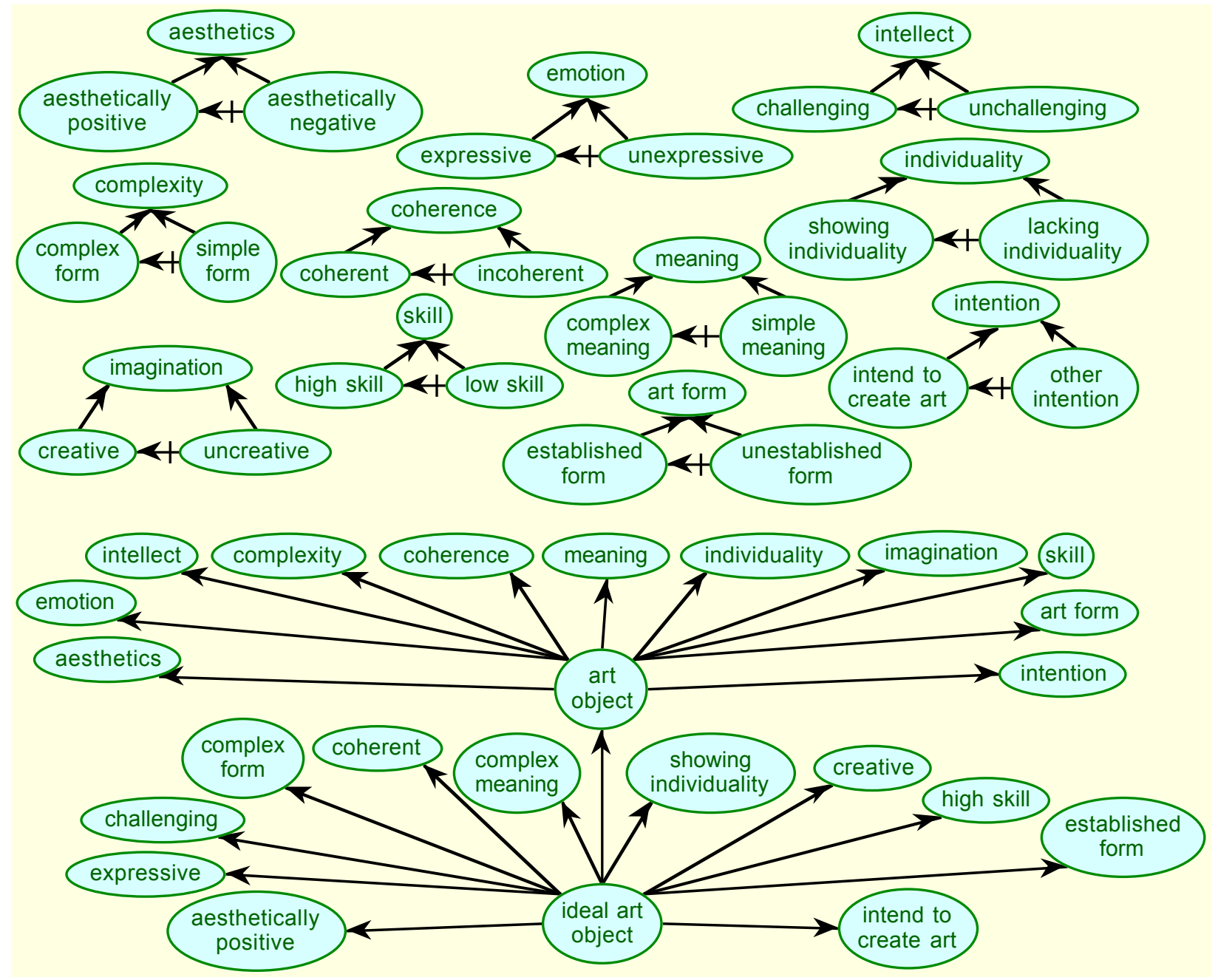

Figure 6 Conceptual dimensions of art objects

The stereotypical ideal art object templet is of interest because, while many art objects conform to the ideal, there are also be many exceptions that are of particular interest just because they exemplify the boundaries of the norm. People will use the templet to construe something as an art object but not, say, challenging or aesthetically positive. One will usually not wish to consider all the $2048\left(2^{11}\right)$ "but not" possibilities, although some of them may become so commonly instantiated as to be given specific names. This potential explosion of "but not" possibilities is one reason why the original cluster concept notion has been criticized (Boër, 
1974), but the dynamic creation of transient templets is an intrinsic capability of a construct network.

Note that the frame templet, art object, is itself the pole of a construct as illustrated in Fig.5. Construing an experience from the perspective of a particular frame is itself a choice of one construct pole rather than another. Note also that the 'worst possible' art object that entails all the negative poles can still be construed as an art object, just a very poor one. We can still choose to fit the templet, perhaps sarcastically.

\subsection{Schema, Faceted Taxonomy, Conceptual Grid, Frame}

The generic structure illustrated in Fig. 6 where the connotations of a templet such as art object are represented by the way it entails the range of convenience templets of a number of relevant constructs has been given many names in the literature.

In the context of human memory processes, Bartlett (1932) terms the network of connotations associated with a templet a schema which is fitted to and modified by experience, with memory being the term we use for the capability to retrieve such schemata.

In the context of library science Ranganathan (1933) terms the network a faceted taxonomy with the ranges of convenience the facets and the networks below them structured them as taxonomies.

In the context of PCP, Kelly calls the matrix of connotation constructs and the elements of experience that fall under them a conceptual grid (p.301-302), a conceptual matrix (Kelly, 1969b), and a repertory grid (p.270) when used to study relationships.

In the context of knowledge representation, Minsky (1974) terms the connotation constructs a frame for the primary templet with the construct ranges of convenience the slots and the poles the values of those slots.

In the context of developmental psychology, Piaget (1985) adopts Bartlett's notion of schema and terms the fitting of schema to experience assimilation, and the elaboration of schema to fit experience accommodation, with the processes competing in a process of cognitive equilibration.

In the context of linguistics Fillmore (1985) argues that frames provide the basis for natural language understanding, and is developing Framenet as a widely available implementation of a frame-based semantic memory (Fillmore, 2003).

In the context of cognitive psychology, Barsalou (1992) proposes that "frames provide the fundamental representation of knowledge in human cognitions," and gives many detailed examples illustrating this assertion.

Thus, there is a rich and diverse literature outside PCP, but largely consistent with it, that may be used in constructivist studies to elaborate Kelly's theoretical psychology.

\subsection{Semantic Networks}

Pioneers of cognitive science, such as Quillian (1967) in his seminal studies of the computer simulation of human memory and meaning processes, cite Kelly's (1955) psychological model as supporting their own developments of semantic network models of human thought and language. Research in cognitive science and artificial intelligence has developed these models in 
great depth, implemented them in powerful computer programs and applied them to a wide range of applications (Lehmann, 1992).

The networks in this paper are examples of the semantic network formalism. The full formalism provides additional graphic structures to provide a shorthand notation for commonly occurring construct structures such as individuals, relations and numeric constraints (Gaines, 2009), and the logical complexity of anticipatory inference with such structures has been studied in depth in the literature on description logics (Baader, Calvanese, McGuinness, Nardi and Patel-Schneider, 2003).

These more elaborate semantic networks are also construct networks as discussed above, and the primary inference mechanisms based on them are those of entailment and contrast, expressed in the literature as one 'concept' subsuming another or being disjoint with another. For example, the cardinality constraints on a family of having zero, one, more than one children, are logical contrasts generating three bipolar constructs.

The evolution of the precise logical/mathematical structures of description logics from the psychological structures of construct networks parallels the evolution of precise, quantitative, scientific models from the imprecise, qualitative, folk science of everyday life. Personal and professional science have a common foundation of anticipation based on experience represented in construct networks.

\section{Anticipation, Experience And Language}

An anticipatory system assimilates its experience to its current templets and, through their entailments and contrasts, anticipates other aspects of those experiences. In doing so it may act to ensure the experience fits the templet, thereby changing the world. If it finds that its anticipations fail it may add additional templets to accommodate the experience by providing a better fit, thereby changing itself.

One can view this as the co-evolution of the system and its environment, noting that the separation between them, the individuation of the system is itself a construct of the system or an observer. One can also see why Kelly (p.302) terms his conceptual grid a "cybernetic model"-it conforms with Ashby's (1946) cybernetic model of the cerebral cortex as an ultrastable system that moves from one equilibrium to another as it interacts with its environment, and to Rosenblueth, Wiener and Bigelow's (1943) cybernetic model of the way in which negative feedback generates anticipatory, future-directed, behavior.

A number of notions that play important roles in many psychological theories appear as epiphenomenal terms for processes of anticipation Kelly's cybernetic model. Learning is a label for elaborations of the construct system inferred to underlie observed activity. Motivation is a label for the choice of templet inferred to underlie observed activity. Internal and external, self and not-self are ways of dichotomously construing a construct network, but it is also possible to bypass the dicotomy and construe the embedded, ecological and collective aspects of construct networks distributed across multiple people and physical artifacts (Gaines, 1994).

\subsection{Representing Experience}

To provide a concrete example Fig.7 shows some aspects of the experience of some of the art objects that Gaut discusses in relation to his art object templet. Experiences of art objects 
generate templets that entail the templet art object, poles of the constructs whose ranges of convenience it entails, and other aspects of art objects that may relate to these constructs such as the artist who intended to create the art, or to other templets such as those for objects in a building or physical objects in general.

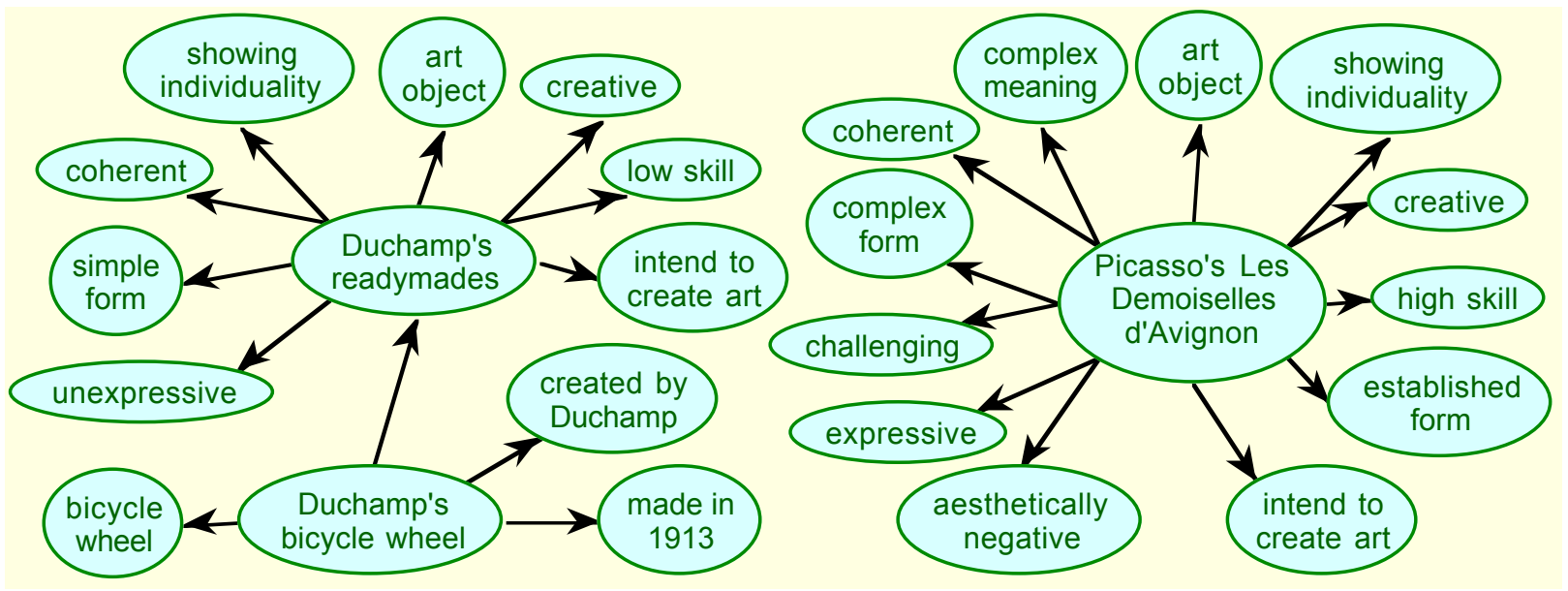

Figure 7 Some art object experiences

Duchamp's (Duve, 1991) submission of everyday, readymade items to art exhibitions is presented by Gaut as a case instantiating several of the negative poles of the art object templet, and perhaps not being construable on some of its entailed constructs. His submissions were sometimes controversial whether accepted or rejected and sometimes accepted virtually unnoticed, corresponding to alternative choices by different people. They are now treated as art objects having been accommodated with a further templet entailing art object but clearly distinct from ideal art object. Picasso's Les Demoiselles d'Avignon is presented by Gaut as an art object that was provocatively aesthetically negative. This may be accommodated by another additional templet or by changing one's notion of aesthetically positive.

We have added a templet for Duchamp's first readymade, the bicycle wheel, to illustrate some aspects of the templet of an individuated experience, that is, one not of a class concept (p.63) such as Duchamp's readymades, but of a countable instance of that class. As Kelly (p.54) notes "counting makes sense if the things are distinguishable from each other" and "what we count depends on what we abstract to be counted." Individuation corresponds to unicity, that the derivations of the templet only fit one particular individual.

Individuation is determined by our construct network, and we do not need an additional structure to represent individuals. Note, however, that we often create unique labels to provide distinguishing entailments for experience templets that we choose to construe as individuals. Thus, elements of experience may be abstract summaries generated by many similar experiences or concrete instances of specific experiences, but they are all templets with similar structures within our construct networks.

\subsection{Lexical Entailments}

Note that the names shown for the templets involve no additional structure. They are lexical labels entailed by the templets, shown for purposes of communication as the names of the templets. They may be generated as a lexical description of some of the other entailments of the 
templet, possibly intended as an individuating definite description in Russell's (1905) terminology. However, they may also stand alone as an opaque entailment of the templet.

For example, if one read a novel in which an item in a house was described as "one of Duchamp's readymade" one's construct network might accommodate this with a templet for Duchamp's readymades without any connotations other than they are mentioned in the novel and can be an item in a house. One might conjecture that Duchamp is the name of a company manufacturing kitchen appliances, and only many year later, if at all, find out that readymades are art objects and, even later, that they are somewhat peculiar ones.

As Kelly (p.51) notes, construing does not necessary involve language. Perceptual templets with no lexical entailment may be activated to fit experience, and further templets entailing these may be identified by their entailments without involving any verbalization. Words and phrases are attached to templets as entailed lexical templets through the same processes that lead to other entailments/contrasts in the network.

\section{Conceptual Grid/Matrix Representation of a Construct Network}

We have focused so far on modeling the logical structure of Kelly's psychology in terms of construct networks of templets linked by relations of entailment and contrast. This is the most perspicuous way of visualizing the nature of constructs and the relations discussed in Kelly's organization (p.56-59) and dichotomy (p.59-64) corollaries, and it is supported computationally by semantic network tools.

However, in PCP studies construct networks are also commonly represented by an equivalent model in matrix form which, as discussed in Section 4.3, Kelly terms a conceptual grid/matrix and which is nowadays usually termed a repertory grid. Other chapters in this book cover a wide range of issues in repertory grid elicitation, analysis and interpretation. In this section we comment on a few important issues that arise out of the previous sections.

We will focus on how the methodological principles developed in Section 2 can be addressed in the design and application of computer tools intended to support constructivist studies, in general, how the tools can be used to enhance anticipatory and associated meaning making processes.

\subsection{An Example of Grid Representation}

Fig.8 shows a conceptual grid/matrix, or repertory grid, representation of the art object templet from Figs.6 and 7 with poles of the constructs defining the rows, the elements of experience defining the columns, and the ratings on a one to five scale indicating how Gaut chooses to construe an element on a construct. A 1 to 5 rating scale has been used with 1 for the left-hand pole and 5 for the right although, obviously, any other rating scale would be equally adequate. The meta-value 'any' is represented by an asterisk Use of this meta-value characterizes abstract elements of experience, templets that represent a class of experiences. 
Display 'art object templet' (Gaut, 2000)

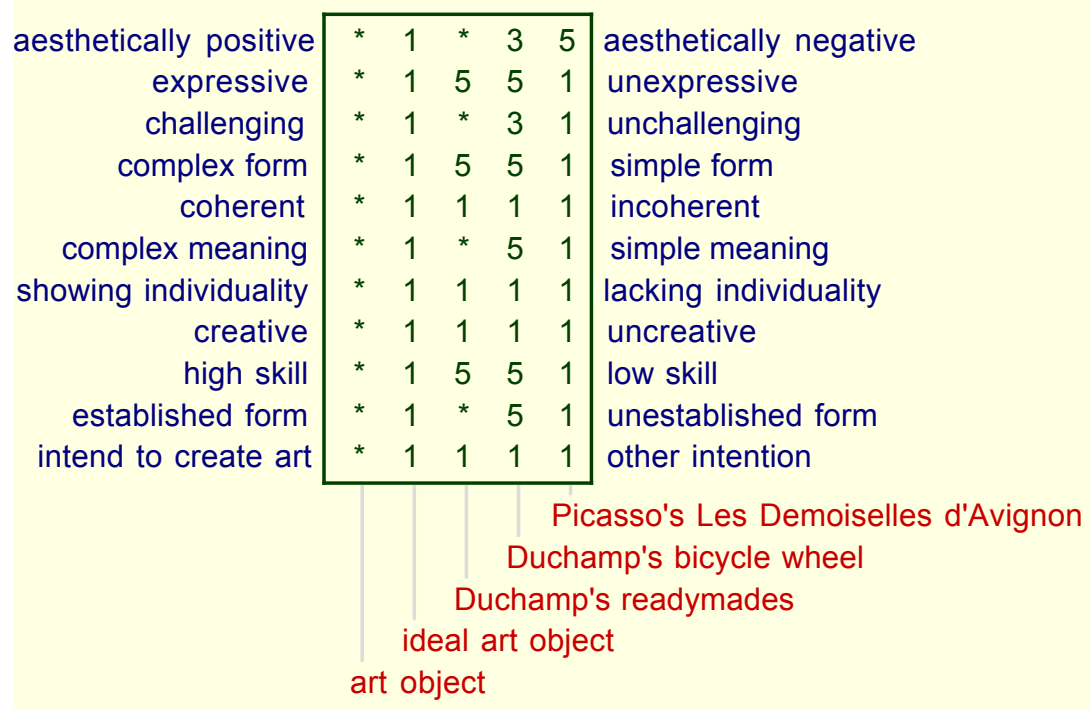

Figure 8 Conceptual grid for art object templet

The grid of Fig.8 is isomorphic to the network of Figs. 6 and 7. We can readily create one representation from the other. The meta-value 'any' represented by '*' enables us to infer that all the other element templets entail the art object templet, and that Duchamp's bicycle wheel templet entails Duchamp's readymades templet.

We can 'flatten' any hierarchical construct network into a repertory grid isomorphic to it, and reconstruct the network from the grid. Construct networks representations make it easy to see how "the personal construct system can be viewed cybernetically" (p.146) as a system for anticipation based on experience. Kelly's isomorphic representation of the personal construct system as a conceptual grid provides the same "cybernetic model" (p.302).

From a computational perspective the network and grid representations carry the same information and can be used equivalently to model human psychological processes. From a human perspective, sometimes one representation is easier to develop and understand than the other. Both should be available as tools for constructivist studies.

\subsection{Hierarchies Generated by Clustering Algorithms}

One approach to the analysis of constructs networks represented in grid form has been to cluster the elements and constructs hierarchically using Shaw's (1980) Focus clustering algorithm. A reasonable methodological question is what relation the hierarchies produced have to those in the network representation. Fig.9 shows the home furnishing network of Fig.5 with individual templets instantiating all contrasting templets, and Fig.10 shows its representation in grid form, again arbitrarily using a 1 to 5 rating scale with 1 representing a left-hand pole. The meta-value 'inapplicable' represented by ' $\sim$ ' is now needed since some elements are not within the range of convenience of some constructs. 


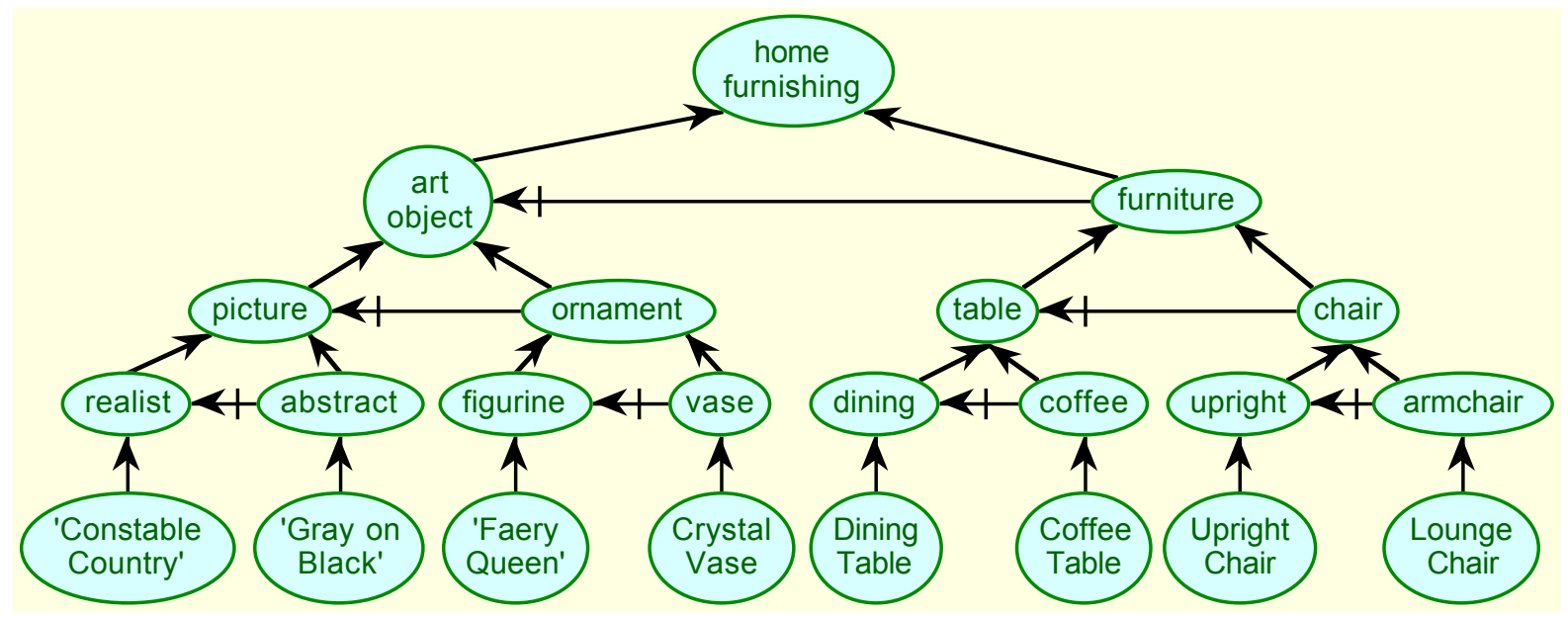

Figure 9 Home furnishing network with individual templets

Display 'home furnishing frame'

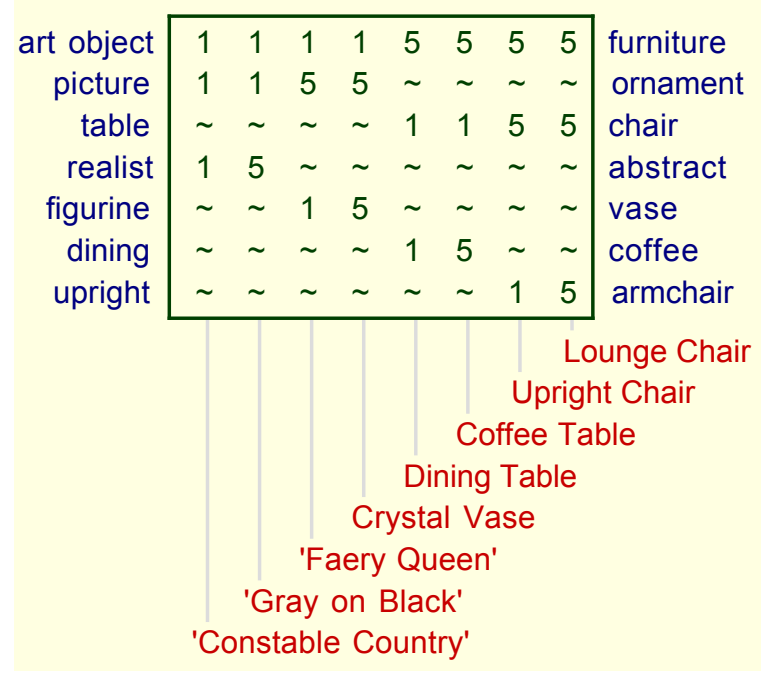

Figure 10 Grid representation of home furnishing network

Focus sorts the rows and columns of the grid to bring similar items together, and represents the degree of match as a hierarchical cluster as shown in Fig.11. It can be seen at the bottom right that the hierarchical structure of the network representation of Fig.9 has been regenerated by the Focus matching and sorting algorithms.

An important feature of the Focus analysis is the presentation of the clusters in terms of the sorted grid. For example, if we examine the cluster, 'Faery Queen' and Crystal Vase, we can see that these elements are integrated by the constructs art object-furniture and picture-ornament, and differentiated by the construct figurine-vase. The Focus clusters can provide input to the meaning processes of those with an interest in the grid because the meaning of the clusters can be understood in terms of the original grid data. 


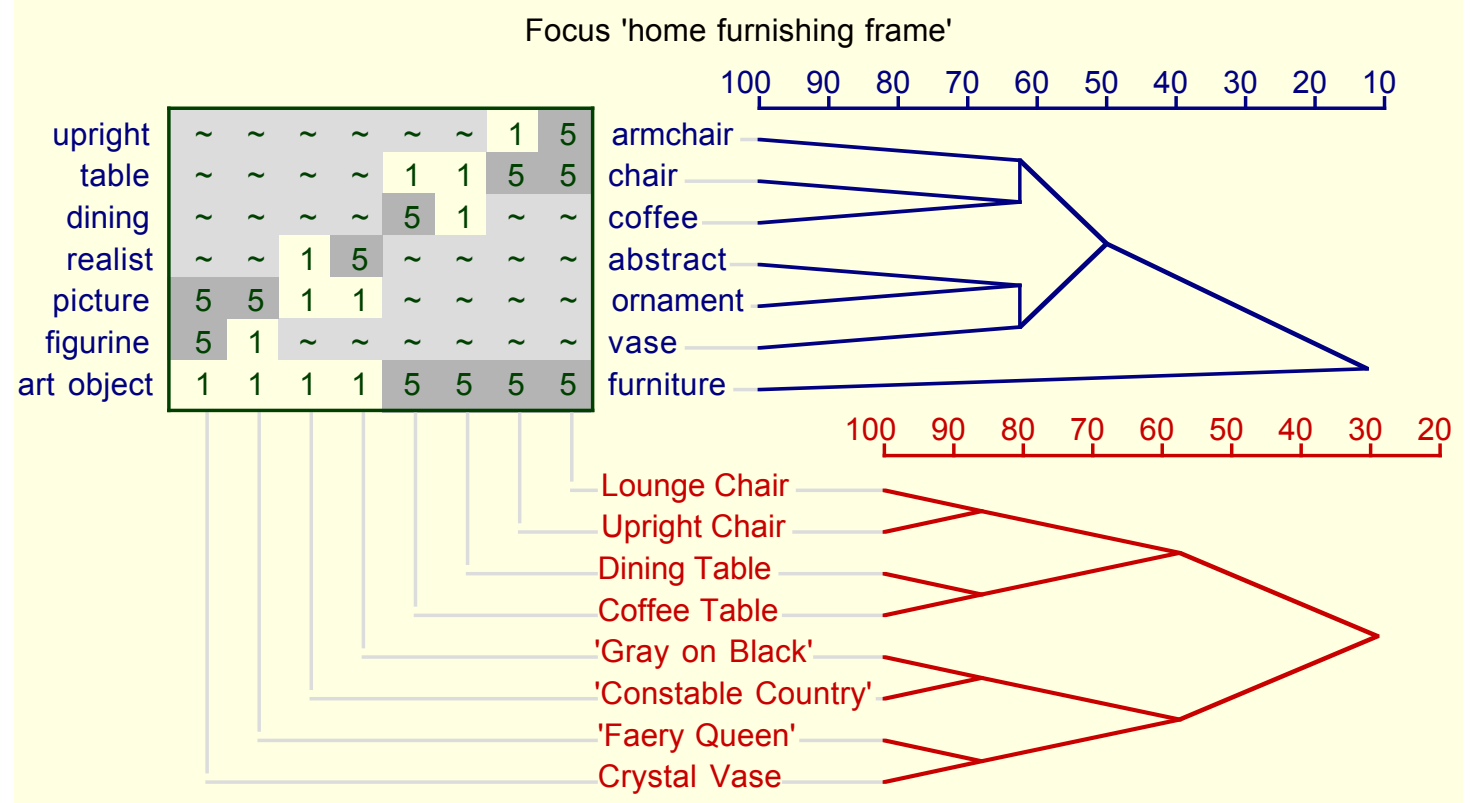

Figure 11 Focus analysis of grid representation of home furnishing frame

Note the importance of supporting the meta-value 'inapplicable' in allowing constructs whose range of convenience does not encompass all the elements to be represented in grid form. Elicitation procedures that force the use of constructs that encompass all the elements can fail to elicit significant constructs. For example, consider a grid for the frame holiday resorts where some resorts are by an ocean or lake and others are inland. The construct has sandy beacheshas stony beaches may be important to the anticipation of the relative attractiveness of the resorts to which it is applicable but irrelevant to others.

\subsection{Enhancing The Meaning Of Analyses Through User Interaction}

Constructivist theory emphasizes the importance of our interactions with the world in coming to make it meaningful. Making analyses interactive to allow their meanings to be explored has been a major objective of our research.

For example, when a grid is being elicited in the WebGrid component of Rep 5, users can analyze it at any time to guide the elicitation. If they feel that a surprising feature of the analysis results from a misconstruction they have entered they can adjust this simply and easily and see the impact on the analysis by clicking on an element or construct to go to the rating system, adjust the entered construction, and see the impact on the analysis.

They can also switch to a mode where if they click on an element or construct that item is temporarily removed from the analysis so that they can see how sensitive the analysis is to the presence of that element or construct. Figure 12 shows a Focus analysis of a simple grid on learning situations. The user may notice that the construct equipment-no equipment is an outlier and wonder what effect it is having on the element clustering. Clicking on this construct excludes it from the analysis, producing the new analysis shown in Fig.13, where it can be seen, for example, that film and lecture are now more tightly clustered. This may cause the user to question whether the involvement of equipment is relevant to their evaluation of learning situations. 


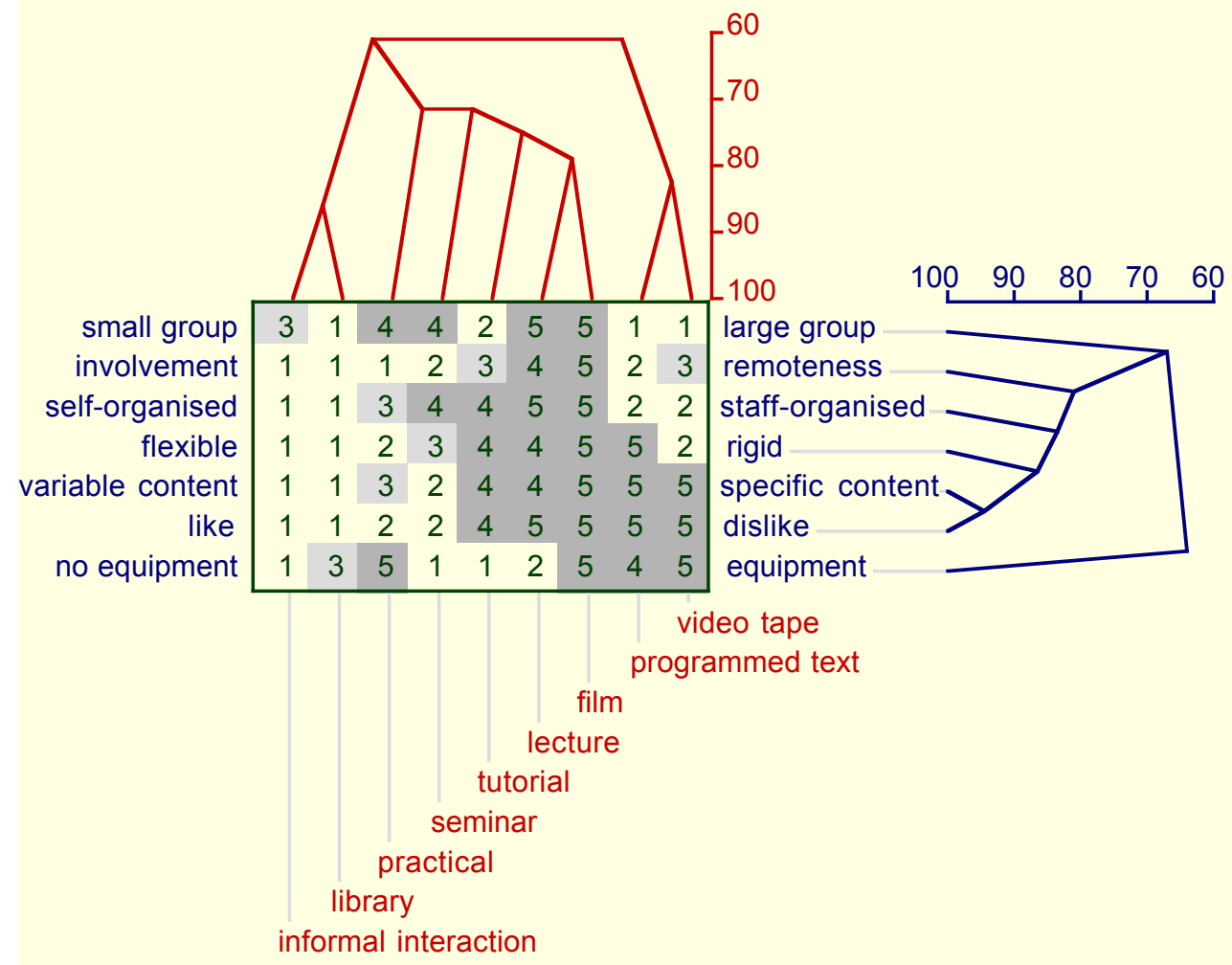

Figure 12 Focus analysis of grid on learning situations

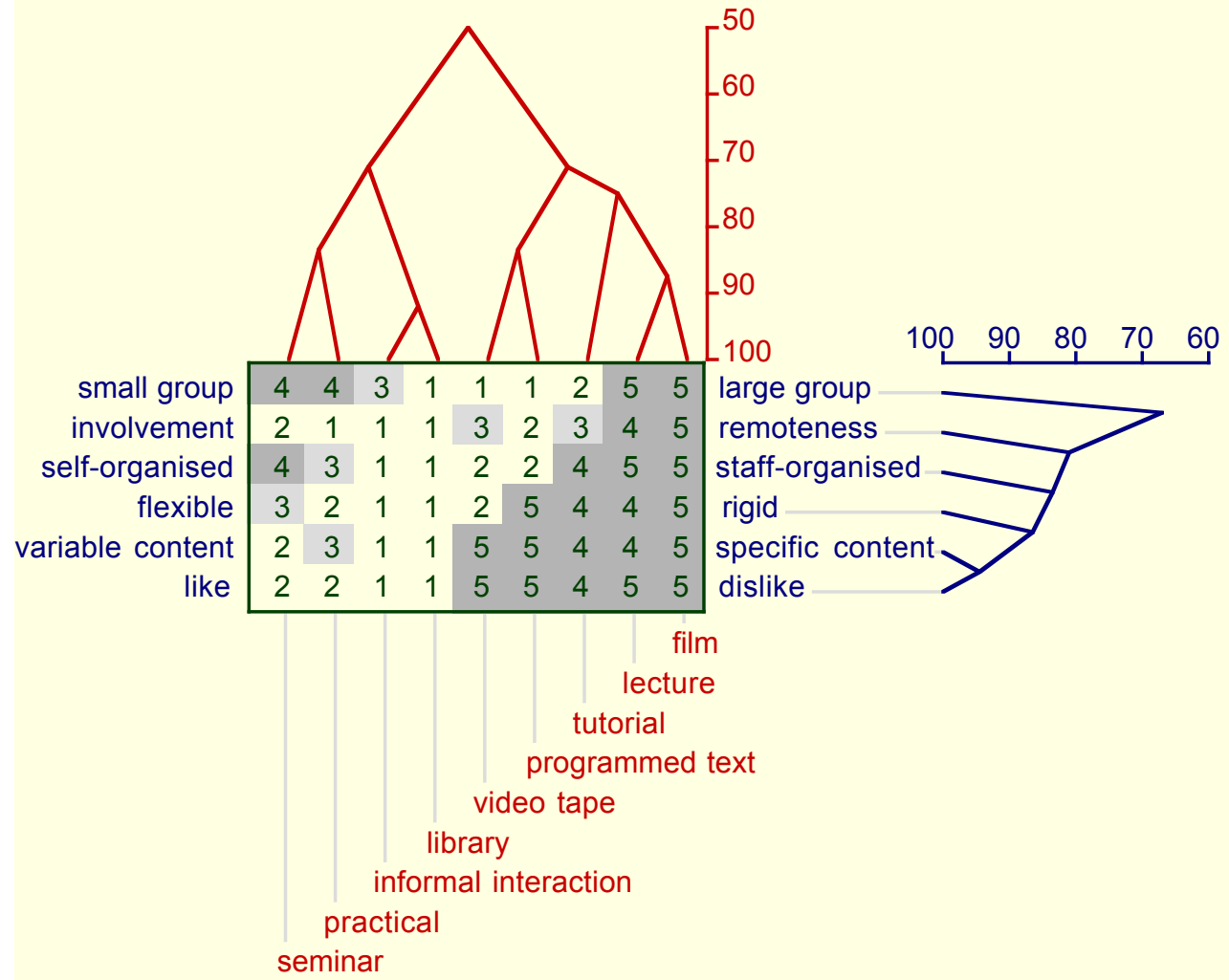

Figure 13 Focus analysis of grid on learning situations with a construct excluded 
What is important is that users are able to explore the anticipatory consequences of various components of their construct systems and thereby come to understand them better, that is, to enhance their meaning. Modern computers can process the data at a rate which matches the speed of the users' thought processes, and the analyses they produce are no longer opaque and authoritarian but interactive and responsive to the users explorations of their construct systems.

\subsection{Understanding and Exploring Psychological Space}

Kelly (ch.6) introduces a compelling geometric metaphor for a construct system, that the constructs are the axes of a multi-dimensional psychological space, and the construed elements of experience are points in this space. Gower (1966) showed that principal components analysis could be construed not as an opaque statistical technique but as a rotation of data points in the multi-dimensional space of the variables used to represent them. Slater $(1976 ; 1977)$ used Kelly's and Gower's insights to develop his Ingrid program which used principal components analysis to present a grid as a two-dimensional map.

One problem of principal components analysis is that, unlike the Focus cluster analysis, there is no link to the grid data and hence the user may have difficulty in understanding the meaning of the output. We have addressed this issue in two ways: by providing the same interactivity as for Focus, making it easy to exclude one or more elements or constructs from the analysis and construe the impact of doing so; and by providing a 2- or 3-D cross-plot capability that makes the geometric metaphor clear. For example Fig.14 shows a crossplot of the elements from the grid on learning situations on three of the constructs.

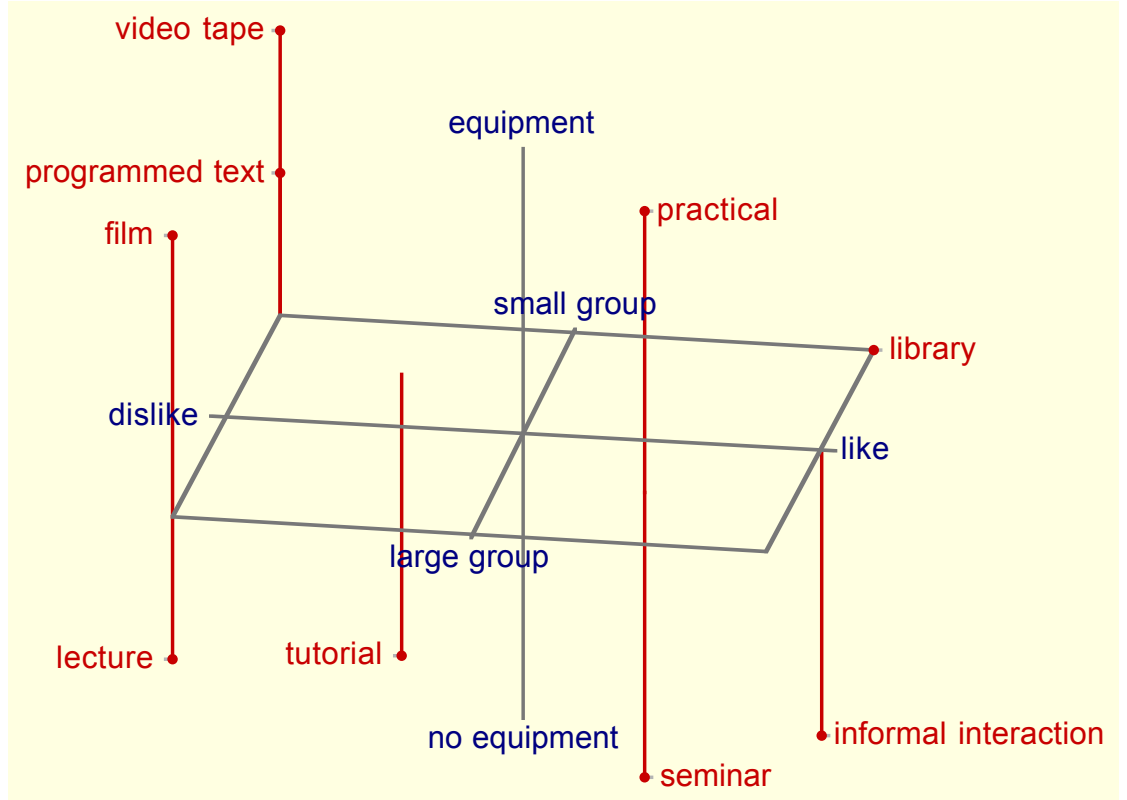

Figure 14 Crossplot of three constructs from grid on learning situations

Crossplots are a useful capability as they are commonly used to display how elements relate to particular constructs, but the application here is to help the user understand the psychological space metaphor in preparation for understanding a principal components analysis (Fig.15) as a rotation of that space. Again, users can click on any element or construct in Fig.15 to exclude it from the analysis, and explore its role in their construct system. 


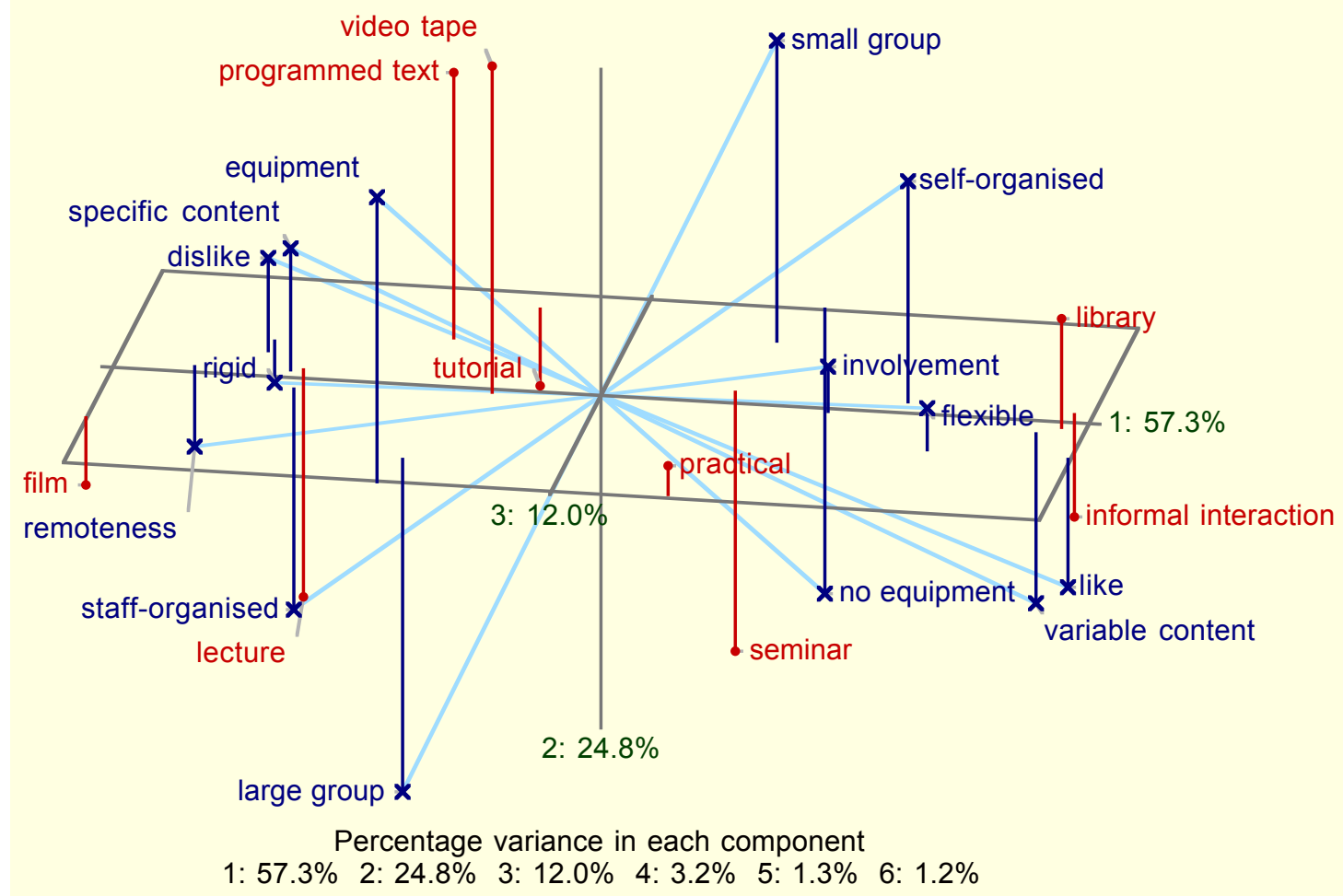

Figure 15 Principal components analysis of grid on learning situations

Interactivity supporting explorations of meaning is one of the most important capabilities of computer systems. As an outcome of their interactions with the analyses, users come to reconstrue them, not as definitive representations of their construct systems but rather as flexible interpretations sensitive to the elements, constructs and ratings that the user provide. Coming to understand this sensitivity not only enhances the meanings of the analyses but also provides insights into similar sensitivities in their own and others construct systems.

\subsection{Multiple Grid Analysis}

As discussed in Section 2, individuals are concerned with calibrating their personal meanings against those of others, and the comparison of construct networks is an important capability to support with computer tools. The techniques for doing this are very effective but involve strong assumptions as discussed in methodological principles 2 and 3, and it is important that these assumptions be made explicit and investigated.

Consider the exchange grid situation where we elicit a grid from one person and then have a second person fill in the ratings on 'the same' elements and constructs. It is simple to compute the differences in ratings between the two grids, sort the matches and plot them to show areas of agreement and disagreement as shown in Fig.16. However, the source of the differences is not, and cannot, be determined from this analysis. Is video tape being construed very differently because one person envisions its use to show prepared materials and the other to allow students to capture their own experiences, or do they both envision prepared materials and one sees tape allowing more flexible and self-organized use than film because it can more readily be accessed by individuals, or is there a general difference in the way in which the terms flexible and rigid are being used? 


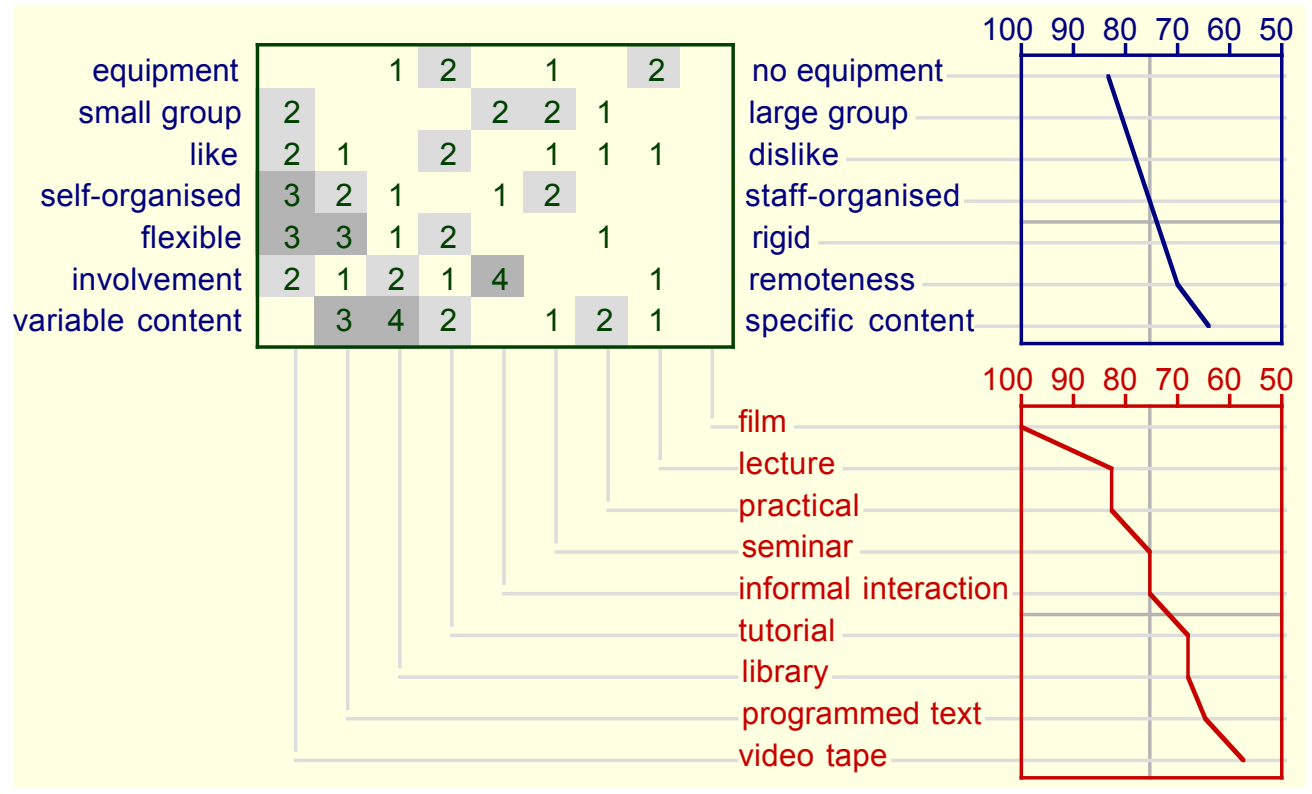

\section{Figure 16 Comparison of two grids with 'the same' elements and constructs}

These various possibilities are fruitful sources of discussion between those from whom the grids were elicited, and only they can resolve them. The realization that they are using the same terms with different meanings may be the most important outcome for the people involved. By using the techniques already described of excluding elements and constructs from the analysis they may also come to understand how the apparent 'sameness' of elements or constructs is dependent on the constructs, or elements, respectively used to compare them.

Principal components analysis of the composite grids produced on the assumption that the elements are 'the same' (Fig.17) or the constructs are 'the same' (Fig.18) can trigger similar insights. The relative rotations of construct axes with the same pole names in Fig.17 corresponds to the relative construct matches in Fig. 16 but provides an alternative visualization. The relative positions of the elements in the compound psychological space of Fig.18 corresponds to the relative elements matches in Fig.16 but provides another alternative visualization.

However, the most insightful realization is that these two analyses are using exactly the same construct network data but making two different assumptions, either that there is a common usage of the element terms or a common usage of the construct terms. If different individuals construe the same experiences differently is it because they having different experiences or because they use the constructs idiosyncratically? If the same individual construes the same entities differently at a later time is it because the entities have changed or because their construct system has changed? There is a fundamental uncertainty underlying such questions but it is often resolved tacitly without the realization that there are other choices. 


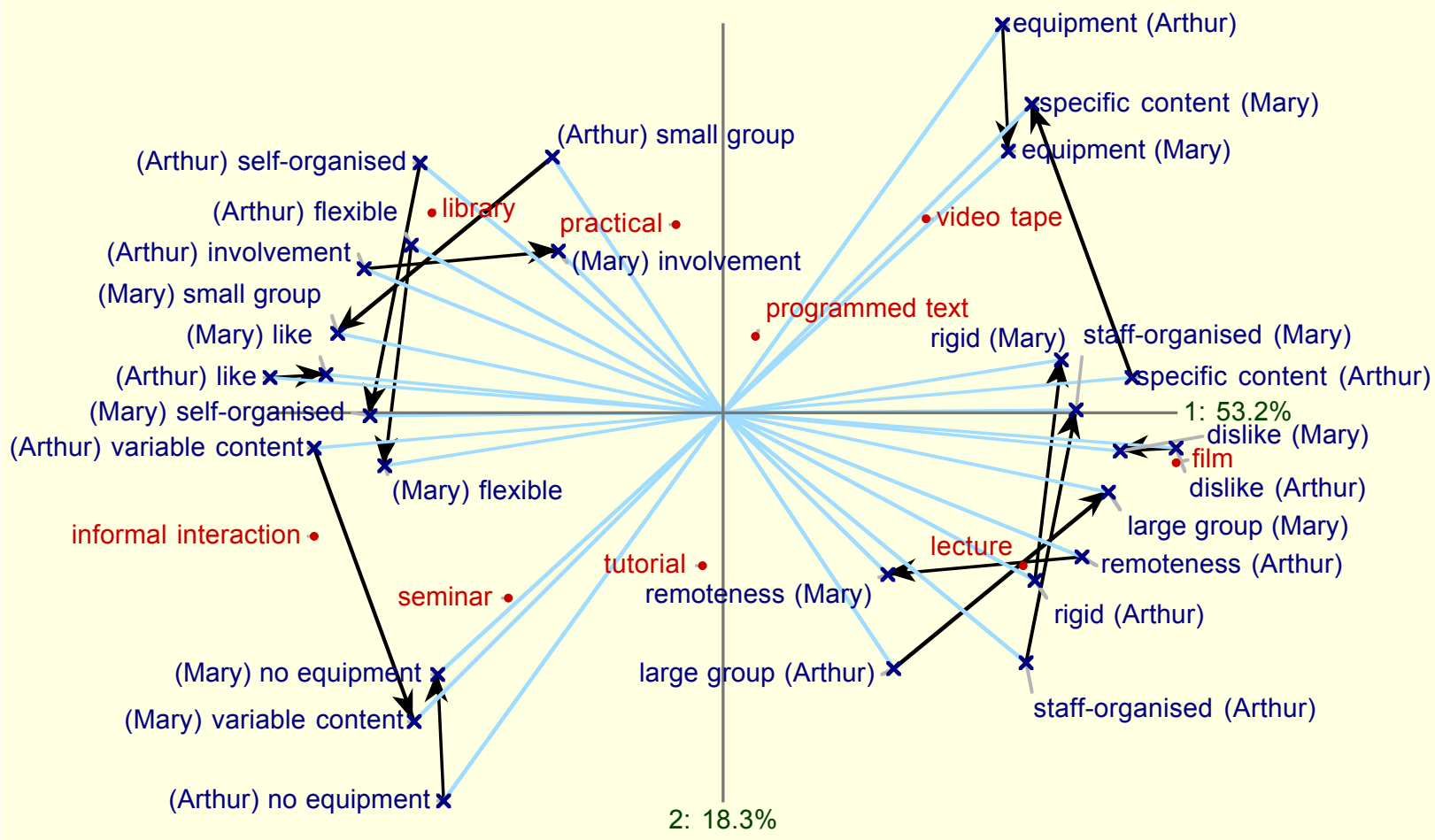

Figure 17 PrinGrid analysis of composite of two grids assuming elements are 'the same'

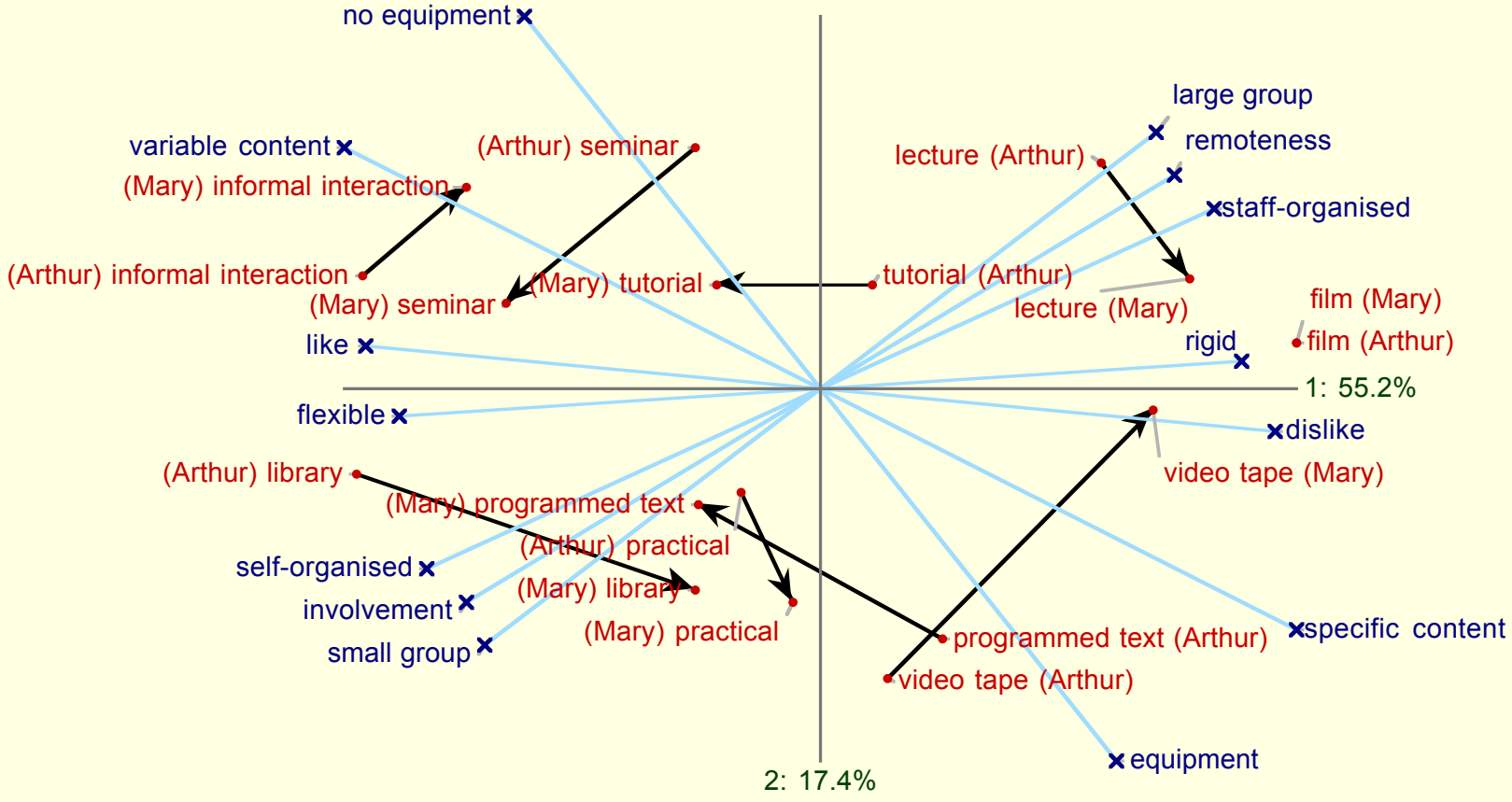

Figure 18 PrinGrid analysis of composite of two grids assuming constructs are 'the same'

Similar considerations apply to the matching of constructs from different people construing the 'same elements' in order to investigate the use of different terms for the same distinction (Shaw and Gaines, 1989). If we assume two grids contain constructs based on construing the same elements it is simple to compute the best match in the second grid for each construct in the first 
grid and display it as shown in Figure 19. If all the constructs have reasonably good matches then the two people can anticipate the distinctions the other will make, even if the terms they use are different, and hence they may have a meaningful social interaction in the domain under consideration-Kelly's sociality corollary (p.95). The element plot indicates that, notwithstanding this mutual construing, they may still have some difficulty communicating about some of the elements.

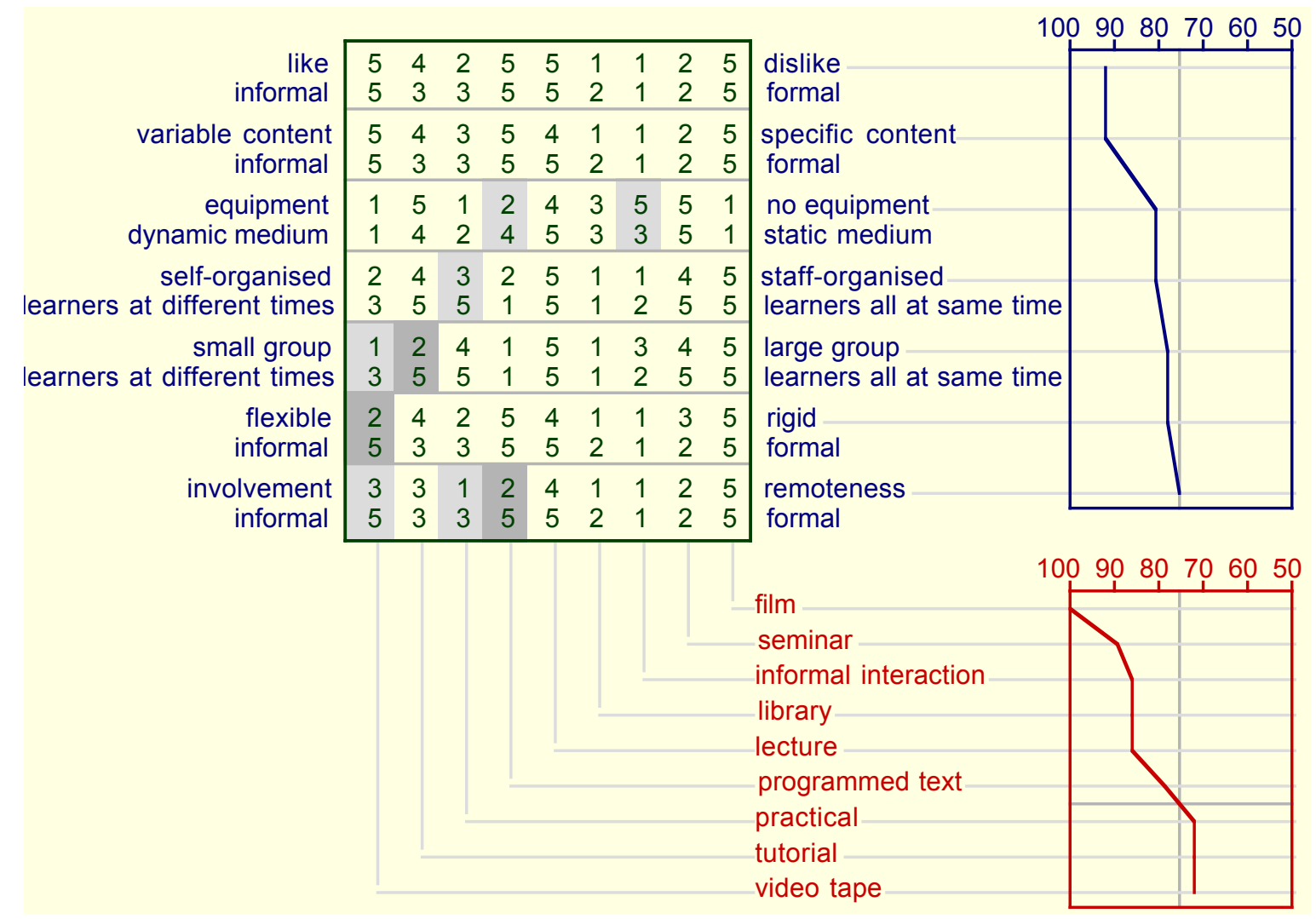

Figure 17 Comparison of two grids with 'the same' elements to match constructs

The same considerations apply to the analysis of sets of grids. Shaw's (1980) use in her SocioGrids algorithms of what have come to been called Procrustean analysis techniques (Gower and Dijksterhuis, 2004) to select what construct in one grid can be best fitted to one in another grid construing the same elements is meaningful only to the extent that the elements are in some sense 'the same.' Shaw's technique for the extraction of a consensus construct network from multiple grids by first selecting best matches and then filtering out those that have the best average match across all grids creates a consensus or mode grid that is robust against some degree of variation in the meaning of the supposedly 'same' elements. Experimenting with excluding grids that are outliers in the socionets produced can provide insights into the factors affecting the consensus. The product being a mode grid of actual constructs that can be traced back to their sources makes the output more meaningful than the statistically constructed grids produced by a general-purpose Procrustes analysis package not targeted on grid data.

It should be noted that these are not issues created by the use of computer programs. The problem of cross-calibrating meanings is a fundamental one for any human community, and the issues faced in providing computer support just make explicit what is usually tacit. The value of 
computer tools is not that they 'solve' the problem, but that they make explicit issues of communication and provide a framework in which to discuss them and investigate their sources and impact. Discussion by those involved supported by exploratory re-analyses with computer tools can make more meaningful the communication processes of a group of people with common interests.

These issues of commonality of meaning are also important for those conducting studies of the construct systems of others since, if they attempt to interpret the networks or grids themselves, they are putting themselves in the exchange situation, attempting to match the terminology of others to their own. Even when elements are thought to be highly public, such as well-known products in a market research survey, it is possible for subjects to be thinking of different aspects of their use, and even when the terms for construct poles appear to be obvious colloquial phrases the intended meaning may not be at all transparent.

\section{Conclusions}

We have attempted in this chapter to provide bridges between Kelly's formulation of personal construct psychology and its representation in semantic networks and conceptual grids. After having spent over thirty years developing and making available computer-based tools to support personal construct psychology, we are concerned that these tools are readily detached from their roots and used outside a constructivist context. They are easily used and produce impressive output, and yet the effectiveness of that use and the interpretation of that output is highly dependent on those using them adopting a constructivist stance to all aspects of the activity.

This includes everyone involved in the study. End-users of PCP tools should not be construed as 'subjects' in the classical sense of the word but as fellow human beings engaged in elaborating their meaning systems in the ways that we all do, and cannot avoid, or be prevented from doing. There is, and should be, a payback from being part of a grid elicitation and interpretation process with the currency being new personal insights, reflections on one's construct system, its origins, relations to those of others, and so on.

Constructivist studies must factor this into their design, that they do not leave their 'subjects' unchanged, and that the 'data collected' is a snapshot of a process not a static measurement of a stable system. This is the psychological equivalent of the Heisenberg uncertainty principle in quantum physics, that the 'observer' cannot leave the system unchanged. It is an effect in most investigations of human activity, but it is a particularly profound effect when the tools used intrinsically provoke reflection on ones personal construct system.

Returning to the methodological issues of Section 2, if we had one grand recommendation for addressing all of them it would be to apply constructivist methods to your entire study, not just use them within the study itself. We have used constructivist methods supported by computer tools to facilitate the creative processes of research students (Shaw, 1994; Shaw and Gaines, 1998) and professional research teams (Gaines and Shaw, 1994; Gaines, 2003) in a wide range of disciplines. Your own construct network, those of your collaborators, those of the disciplinary area, those of assessors such as referees, examiners, journal editors, journal readers, funding agencies, and so on, all play important roles in the study. Studying them using the methods you will be using in the study can have a major impact in focusing the study, making more effective use of resources, and achieving more with greater value. 
We have also found concept mapping and semantic network tools to be complementary to repertory grid tools and generally use both in most studies, moving freely back and forth between them to engage those involved in the study as much and as fluidly as possible. We see less use of network representations in PCP studies than is appropriate, and hope that this article encourages their more widespread use.

\section{Acknowledgements}

We are grateful to Bob Green for his perceptive comments which helped us improve this article.

\section{Access To Software and Related Articles}

WebGrid is available at http://gigi.cpsc.ucalgary.ca:2000

Rep 5 is available at http://repgrid.com

Relevant papers and reports are available http://cpsc.ucalgary.ca/ gaines/reports/

\section{References}

Ashby, W. R. (1946). Dynamics of the cerebral cortex: the behavioural properties of systems in equilibrium. American Journal of Psychology 59, 682-686.

Baader, F., Calvanese, D., McGuinness, D., Nardi, D. and Patel-Schneider, P., eds. (2003). The Description Logic Handbook. Cambridge University Press, Cambridge.

Barsalou, L. W. (1992). Frames, concepts and conceptual fields. In Frames, Fields, and Contrasts: New Essays in Semantic and Lexical Organization Lehrer, A. and Kittay, E. F., eds., pp. 21-74. L. Erlbaum, Hillsdale, N.J.

Bartlett, F. C. (1932). Remembering: A Study in Experimental and Social Psychology. Cambridge University Press, Cambridge.

Boër, S. E. (1974). Cluster-concepts and sufficiency definitions. Philosophical Studies 26, 119125.

Boisvert, R. D. (1988). Dewey's Metaphysics. Fordham University Press, New York.

Boose, J. H. (1984). Personal construct theory and the transfer of human expertise. In Proceedings AAAI-84, pp. 27-33. American Association for Artificial Intelligence, Menlo Park, CA.

Colby, K. M. (1981). Modeling a paranoid mind. Behavioral and Brain Sciences 4, 515-560.

Collins, A. M. and Quillian, M. R. (1969). Retrieval time from semantic memory. Journal of Verbal Learning and Verbal Behavior 8, 240-247.

Danto, A. (1964). The artworld. Journal of Philosophy 61, 571-584.

Davis, M., Logemann, G. and Loveland, D. (1962). A machine program for theorem proving. Communications of the ACM 5, 394-397.

Dewey, J. (1911). Causation. In A Cyclopedia of Education Monroe, P., ed, pp. 381 (Dewey Middle Works 6). The Macmillan company, New York,.

Dewey, J. (1917). The need for a recovery of philosophy. In Creative Intelligence, Essays in the Pragmatic Attitude Dewey, J., ed, pp. 3-69. Henry Holt, New York.

Dewey, J. (1933). How We Think. Heath, New York.

Duve, T. d. (1991). Pictorial Nominalism: On Marcel Duchamp's Passage From Painting To The Readymade. University of Minnesota Press, Minneapolis. 
Fillmore, C. J. (1985). Frames and the semantics of understanding. Quaderni di Semantica 6, 222-254.

Fillmore, C. J. (2003). Double-decker definitions: the role of frames in meaning explanations. Sign Language Studies 3, 263-295.

Gaines, B. R. and Shaw, M. L. G. (1993). Basing knowledge acquisition tools in personal construct psychology. Knowledge Engineering Review 8, 49-85.

Gaines, B. R. (1994). The collective stance in modeling expertise in individuals and organizations. International Journal of Expert Systems 7, 21-51.

Gaines, B. R. and Shaw, M. L. G. (1994). Using knowledge acquisition and representation tools to support scientific communities. In AAAI'94: Proceedings of the Twelfth National Conference on Artificial Intelligence, pp. 707-714. AAAI Press/MIT Press, Menlo Park, California.

Gaines, B. R. (2003). Organizational knowledge acquisition. In Handbook on Knowledge Management: 1 Holsapple, C. W., ed, pp. 317-347. Springer, Berlin.

Gaines, B. R. (2009). Designing visual languages for description logics. Journal of Logic, Language and Information 18, 217-250.

Gaut, B. (2000). "Art" as a cluster concept. In Theories of Art Today Carroll, N., ed, pp. 25-44. University of Wisconsin Press, Madison, WI.

Gaut, B. (2005). The cluster account of art defended. The British Journal of Aesthetics 45, 273-288.

Gower, J. (1966). Some distance properties of latent root and vector methods used in multivariate analysis. Biometrika 53, 325-338.

Gower, J. C. and Dijksterhuis, G. B. (2004). Procrustes Problems. Oxford University Press, Oxford.

Harnad, S. (1990). The symbol grounding problem. Physica D 42, 335-346.

Hull, C. L. (1940). Mathematico-Deductive Theory of Rote Learning: A Study in Scientific Methodology. Yale University Press, New Haven.

Hume, D. (1888). A Treatise of Human Nature. Clarendon Press, Oxford.

Kelly, G. A. (1955). The Psychology of Personal Constructs.

Kelly, G. A. (1963). Aldous: the personable computer. In Computer Simulation of Personality Tomkins, S. S. and Messick, S., eds., pp. 221-229. Wiley, New York.

Kelly, G. A. (1969a). Humanistic methodology in psychological research. Journal of Humanistic Psychology 9, 53-65.

Kelly, G. A. (1969b). A mathematical approach to psychology. In Clinical Psychology and Personality: The Selected Papers of George Kelly Maher, B., ed, pp. 94-113. Wiley, New York.

Kelly, G. A. (1970). A brief introduction to personal construct theory. In Perspectives in Personal Construct Theory Bannister, D., ed, pp. 1-29. Academic Press, London.

Kulp, C. B. (1992). The End of Epistemology: Dewey and His Current Allies on the Spectator Theory of Knowledge. Greenwood, Westport, CN.

Lehmann, F. (1992). Semantic networks. Computers \& Mathematics with Applications 23, 150 .

Minsky, M. (1974). A Framework for Representing Knowledge. MIT-AI Laboratory Memo 306, Boston.

Pecher, D. and Zwaan, R. A. (2005). Grounding Cognition: The Role of Perception And Action In Memory, Language, And Thinking. Cambridge University Press, New York. 
Piaget, J. (1985). The Equilibration of Cognitive Structures: The Central Problem of Intellectual Development. University of Chicago Press, Chicago.

Quastler, H. (1955). Information Theory in Psychology: Problems and Methods. Free Press, Glencoe, IL.

Quillian, M. R. (1967). Word concepts: a theory and simulation of some basic semantic capabilities. Behavioral Science 12, 410-430.

Ranganathan, S. R. (1933). Colon Classification. The Madras Library Association, Madras.

Rorty, R. (1982). Consequences of Pragmatism: Essays, 1972-1980. University of Minnesota Press, Minneapolis.

Rosenblueth, A., Wiener, N. and Bigelow, J. (1943). Behavior, purpose and teleology. Philosophy of Science 10, 18-24.

Russell, B. (1905). On denoting. Mind 14, 479-493.

Shaw, M. L. G. (1978). Interactive computer programs for eliciting personal models of the world. In Personal Construct Psychology 1977 Fransella, F., ed, pp. 59-67. Academic Press, London.

Shaw, M. L. G. (1980). On Becoming a Personal Scientist: Interactive Computer Elicitation of Personal Models of the World. Academic Press, London.

Shaw, M. L. G. and Gaines, B. R. (1983). A computer aid to knowledge engineering. In Proceedings of British Computer Society Conference on Expert Systems, pp. 263271. British Computer Society, Cambridge.

Shaw, M. L. G. and Gaines, B. R. (1989). Comparing conceptual structures: consensus, conflict, correspondence and contrast. Knowledge Acquisition 1, 341-363.

Shaw, M. L. G. (1994). Women, scholarship and information technology: A post-modern perspective. Transactions of the Royal Society of Canada 5, 113-131.

Shaw, M. L. G. and Gaines, B. R. (1998). A research-based masters program in the workplace. Proceedings of WCCCE'98: Western Canadian Conference on Computing Education

Shook, J. R. (2000). Dewey's Empirical Theory of Knowledge and Reality. Vanderbilt University Press, Nashville.

Slater, P., ed. (1976). Dimensions of Intrapersonal Space: Volume 1. John Wiley, London.

Slater, P., ed. (1977). Dimensions of Intrapersonal Space: Volume 2. John Wiley, London.

Taddeo, M. and Floridi, L. (2005). Solving the symbol grounding problem: a critical review of fifteen years of research. Journal of Experimental \& Theoretical Artificial Intelligence 17,419 - 445 .

Weitz, M. (1977). The Opening Mind: A Philosophical Study of Humanistic Concepts. University of Chicago Press, Chicago.

Weizenbaum, J. (1966). ELIZA - a computer program for the study of natural language communication between man and machine. Journal of the ACM 9, 36-45.

Yorke, M. (2001). Bipolarity... or Not? Some Conceptual Problems Relating to Bipolar Rating Scales. British Educational Research Journal 27, 171-186. 